\title{
Own-wage labor supply elasticities: variation across time and estimation methods
}

\author{
Olivier Bargain ${ }^{1,2}$ and Andreas Peich $3^{*}$
}

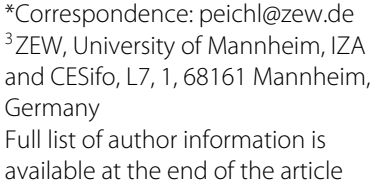

\begin{abstract}
There is a huge variation in the size of labor supply elasticities in the literature, which hampers policy analysis. While recent studies show that preference heterogeneity across countries explains little of this variation, we focus on two other important features: observation period and estimation method. We start with a thorough survey of existing evidence for both Western Europe and the USA, over a long period and from different empirical approaches. Then, our meta-analysis attempts to disentangle the role of time changes and estimation methods. We highlight the key role of time changes, documenting the incredible fall in labor supply elasticities since the 1980s not only for the USA but also in the EU. In contrast, we find no compelling evidence that the choice of estimation method explains variation in elasticity estimates. From our analysis, we derive important guidelines for policy simulations.
\end{abstract}

JEL Classification: C25, C52, H31, J22

Keywords: Household labor supply, Elasticity, Taxation, Europe, USA

\section{Introduction}

Assessing the labor supply responsiveness to tax (and benefit) changes is of key importance for policy analysis-for example, to predict the effect of tax-benefit policy reforms ex ante or to calibrate an optimal tax model. Responsiveness is often summarized by a measure of elasticities or what Chetty et al. (2011) refer to as "steady-state elasticities," i.e., wage or income elasticities of labor market participation or worked hours stemming from a static framework. In principle, these estimates should also provide some information on international differences in labor supply responses. However, the variation in the magnitude of labor supply elasticities found in the literature is huge (see Evers et al. 2008), and there is little agreement among economists on the elasticity size that should be used in economic policy analyses (Bargain et al. 2014; Fuchs et al. 1998) show that only a small share of this variation is driven by genuine differences in work preference across countries. ${ }^{1}$ In fact, other factors account for the large differences in elasticities that are observed across studies: (i) the period of investigation (which may reflect changes in work preferences, for instance) and (ii) modeling choices (estimation method and model specification). To understand the relative contribution of these two factors, a careful and comprehensive survey of the literature on steady-state elasticities is required, which we undertake in the present paper. 
Our survey substantially completes previous reviews on static labor supply models by providing a wider and more comprehensive comparison of international evidence. Handbook studies written in the 1980s mainly focus on estimations using the continuous labor supply model of Hausman (1981) and provide evidence essentially for individuals in couples (Hausman 1985b; Pencavel 1986, for married men, Killingsworth and Heckman 1986, for married women). More recent surveys incorporate some evidence from recent methods (see Blundell and MaCurdy 1999; Meghir and Phillips 2008) or focus on dynamic life-cycle models (Keane 2011; Keane and Rogerson 2012; McClelland and Mok 2012). Yet, most of these surveys mainly summarize the available evidence for the USA and the UK. Evers et al. (2008) suggest a meta-analysis based on estimates for different Western countries but focus essentially on those obtained with the traditional Hausman approach.

We provide a fresh characterization of static labor supply elasticities, collecting old and recent estimates for both Europe and the USA, covering the studies based on the Hausman method, more recent ones based on discrete-choice structural models, and, when available, estimates drawn from natural experiments. We focus on the two margins usually emphasized in the empirical literature on labor supply (Heckman 1993), namely how individuals respond by varying their hours of work (intensive margin) or by deciding whether or not to enter the labor force (extensive margin). ${ }^{2}$ We ignore other margins that are captured in the literature on the elasticity of taxable income (see Meghir and Phillips 2008 and Saez et al. 2012, for surveys) ${ }^{3}$ and leave aside the macroeconomic literature, in which elasticities are often obtained by calibration of general equilibrium models. ${ }^{4}$ We compare 282 elasticity estimates resulting from 92 studies, including 156 wage elasticities for individuals in couples, 70 wage elasticities for single individuals and lone parents, and 56 income elasticities. Our survey broadly confirms the modest consensus reached in the literature, establishing that own-wage elasticities are largest for married women and smaller for men. Recent studies confirm these findings but not the negative elasticities for men as sometimes found in older studies. Estimates for men are generally positive and small, with some exceptions (for instance, Ireland and some German studies). Some of the studies for the USA and the UK, but not all, point to substantial elasticities for single parents while estimates for childless singles are usually missing.

Then, we investigate the relative roles of time changes and estimation techniques in explaining the diversity of estimates found in the literature (which had not been done in previous surveys like Evers et al. 2008). Our meta-analysis, based on both visual displays and meta-regressions, addresses the principal challenge coming from the fact that supposedly overstated estimates due to the Hausman approach coincided with a period characterized by low female participation. Restricting our meta-analysis to years of common support, we find suggestive evidence in favor of the hypothesis of a time decline in elasticities for both the USA and Europe. This confirms and extends the result of Heim (2007) and Blau and Kahn (2007) who show that married women's wage elasticities have strongly declined over time in the USA. A possible explanation for this finding is that a more stable attachment of women to the labor market is responsible for modest participation responses to financial incentives in the recent period. We do not find compelling evidence that the estimation methods matter for the size of elasticities. Rather, the results are indeed driven by time trends.

The rest of the paper is organized as follows. In Section 2, we describe the various empirical approaches to estimate static labor supply elasticities. This is particularly 
important to understand potential biases or differences that may be due to the use of the Hausman approach compared to more recent approaches including discrete-choice model estimations and quasi-experiments. Section 3 surveys estimates of labor supply elasticities in the literature. Section 4 suggests a meta-analysis of the respective contributions of time change and estimation methods. In Section 5, we conclude by discussing the next steps of research and implications for policy analysis.

\section{Estimation methods: a review}

The principal object of examination in this study is the size of wage and income elasticities stemming from static labor supply models. Responsiveness to financial incentives in these models has been identified in various ways. There is no generally agreed-upon standard estimation approach, and we provide here a brief critical review. A more technical and comprehensive presentation of these methods and their identification strategies are provided in Blundell and MaCurdy (1999) and Blundell et al. (2007).

Traditional estimation techniques rely on some functional specification of a labor supply function and the underlying consumption-leisure preferences. Estimation is then made through local linearization of the budget constraint, accounting for the fact that after-tax wages depend on the labor supply choice (Hall 1973) or using more comprehensive techniques (Hausman 1981, 1985a, 1985b). The approach relies on cross-sectional variation in working hours and in the two main covariates, i.e., the after-tax wage and the virtual income (i.e., the intercept of the linearized budget constraint). As a result, the main identification issue is the endogeneity of wages and unearned income, which can be seen as an omitted variable problem. Indeed, wages may be endogenous because unobservables affecting preferences for work, e.g., being a hardworking person, may well be correlated with unobservables affecting productivity and hence wages. Unearned income may be endogenous for similar reasons, i.e., individuals who work harder because of unobserved preferences for work are also likely to have accumulated more assets; if unearned income also represents income from the spouse, positive assortative mating could imply that hardworking individuals will tend to marry similar persons, another reason for the endogeneity issue. Hence, estimates obtained from cross-sectional variation in wages and non-labor income across individuals are potentially biased.

Instrumental variables methods have been suggested, and the validity of the Hausman approach hinges on whether the exclusion assumptions of the economic model hold. Also, estimates are potentially contaminated by measurement errors from the division bias (cf. Ziliak and Kniesner 1999). In addition, a series of practical difficulties limit the application of the Hausman method. First, relying on tangency conditions, the Hausman model is mainly restricted to the case of piecewise linear and convex budget sets, i.e., a partial representation of the effect of tax-benefit policies on household budget constraints. This limitation applies equally to generalizations of the technique to non-parametric estimations (Blomquist and Newey 2002). To account for non-convexities, as in Hausman (1985b) and Hausman and Ruud (1984), labor supply must be specified parametrically together with the corresponding direct utility function, which implies rather restrictive forms for preferences (see the discussion in van Soest and Das 2001). ${ }^{5}$ Second, quasiconcavity of the utility function is implicitly imposed a priori. As discussed by MaCurdy et al. (1990) and MaCurdy (1992), the Hausman method thus requires global satisfaction of the Slutsky condition by the labor supply function for internal consistency of the model, 
an unnecessary behavioral restriction that may bias estimates (see a modern statement in Heim and Meyer 2003 and Meghir and Phillips 2008). ${ }^{6}$ Third, the Hausman model makes it difficult to handle joint labor supply decisions within a couple or participation decisions. Instead of non-participation following simply from the corner solution of the model, fixed costs of work can be introduced, yet this additional source of non-convexity has to be dealt with and results seem to be very sensitive to the model specification (see the discussion in Bourguignon and Magnac 1990).

Instead of estimating a labor supply function, the discrete-choice approach is based on the concept of random utility maximization (see Aaberge et al. 1995, 1999; van Soest 1995; or Hoynes 1996, among others). Thus, it requires the explicit parameterization of consumption-leisure preferences, for utility to be evaluated at each discrete alternative. Tangency conditions need not be imposed, and the model is in principle very general. Labor supply decisions are reduced to choosing among a discrete set of possibilities, e.g., inactivity, part-time, and full-time. This solves several problems encountered with the Hausman method. In particular, modeling includes non-participation as one of the options so that both extensive and intensive margins are directly estimated. The complete effect of the tax-benefit system is easily accounted for, even in the presence of non-convexities in budget sets. Work costs, which also create non-convexities, are dealt with relatively easily. Estimated as model parameters as in Callan et al. (2009) or Blundell et al. (2000), they usually improve the fit of these models as they account for the fact that very few observations exist with a small positive number of worked hours. Very few restrictions on preferences need to be imposed in discrete-choice models, notably because fixed costs of work cannot be disentangled from preference parameters, so that it makes no sense to impose the convexity of preferences (see van Soest et al. 2002; Heim and Meyer 2003; Bargain 2009). The only restriction to the model is the imposition of increasing monotonicity in consumption, which seems a minimum requirement for meaningful interpretation and policy analysis. Joint labor supply decision for couples is a straightforward extension of the basic model in the discrete-choice setting. Yet, many applications still treat husbands' working hours fixed at observed levels and focus on the labor supply of women, i.e., a male chauvinist model (e.g., Bargain 2009; such treatment is typical in Hausman models, e.g., Killingsworth and Heckman 1986). The implication of such separable treatment of spouses' labor supply choices is relatively unknown.

In the discrete-choice approach, identification is mainly provided by non-linearities, non-convexities, and discontinuities in the budget constraint due to tax-benefit rules (see the discussion in Blundell et al. 2007 and Bargain et al. 2014). Precisely, individuals with the same gross wage usually receive different net wages. Indeed, as they are characterized by different circumstances (different marital status, age, family compositions, home-ownership status, disability status) or levels of non-labor income, their effective tax schedules are different, i.e., different actual marginal tax rates or benefit withdrawal rates. Arguably, some of the conditioning characteristics (age, children) are also included as preference variables in the model so that identification is essentially parametric. In practice, some exclusion restrictions come naturally. Indeed, tax-benefit rules depend on characteristics which are much more detailed than usual taste shifters (e.g., benefit rules depending on detailed geographical information while preferences are assumed to depend only on urban versus rural areas or on whether the household lives in the capital city). Additionally, more convincing sources of exogenous variation are also used in some 
studies. Closer to the natural experiment method, these consist in time or regional variation in tax-benefit rules. For instance, in the USA, variation in income tax rules or in the parameters of the Earned Income Tax Credit (EITC) across states is used in Eissa and Hoynes (2004) or Hoynes (1996). Time variation in tax-benefit rules also provides a better identification when policy reforms occur over the period under consideration, as discussed, e.g., in Bargain et al. (2014).

A third approach consists in using policy reforms explicitly in order to identify labor supply responses, without attempting to estimate a structural model (e.g., Eissa and Liebman 1996). Natural experiments based on important tax-benefit reforms in the USA and the UK have been extensively used to identify behavioral parameters (see the survey of Hotz and Scholz 2003, for the USA). For example, Eissa and Liebman (1996) use a difference-in-difference approach to identify the impact of the EITC reforms on the labor supply of single mothers. They find compelling evidence that single mothers joined the labor market in response to increased financial incentives to work. Regarding identification, the definition of control groups might be an issue in difference-in-difference approaches. For instance, responses to EITC expansions affecting single mothers were evaluated using childless women as control group, which may not be ideal given different long-term trends in labor supply in the two groups (see Hotz and Scholz 2003). ${ }^{7}$ Regression discontinuity (RD) is deemed better in this respect since the nature of individuals on both sides of the discontinuity is "as good as random" (cf. Lemieux and Milligan 2008). Overall, much of the evidence is concentrated in studies from the USA, Canada, and the UK. There is less evidence for other countries and notably for continental Europe maybe because large reforms, creating exogenous variation in tax-benefit rules, were less available. Partly for this reason, structural models described above have been very much in use. ${ }^{8}$ The timing of response to policy reforms or policy discontinuity is unclear. Nonetheless, the implicit model that analysts have in mind when discussing the "nextmorning" effect of the policy impact is often a static one (cf. Lemieux and Milligan 2008 or Bargain and Doorley 2011). Reduced-form approaches, based on policy reforms or discontinuities, are increasingly used because natural experiments probably offer one of the most credible sources of identification, despite the limitations outlined above. In this way, it is important to compare estimates from these studies with those stemming from structural model estimations. Unfortunately, these studies do not systematically report wage elasticities. They rather report labor supply elasticities to benefit or tax rate changes. Thus, for comparability purposes, we could include only a few of them in the present survey. Also, the fact that actual reforms-notably welfare reforms in the USA and the UK-typically affect couples or single women with children makes that very little evidence is available for other demographic groups, in particular for childless single individuals.

Finally, a few studies rely on long-term changes in wages as well as on observation grouping in order to address endogeneity and the problem of measurement error in hourly wages discussed above (Devereux 2003, 2004). Blundell et al. (1998) also use taxbenefit policy variation over a long period to identify labor supply responses in the UK using a grouping IV estimator. Long-term variation may pose the problem of assuming that preferences remain stable in the long run, an issue which is rarely discussed. We include most of these studies, at least those for which estimates can be compared with other studies, in our survey. 


\section{Static labor supply elasticities: a survey}

We present here existing evidence on labor supply elasticities for European couples (Table 1), European single individuals (Table 2), and all demographic groups in the USA (Table 3). The reason for this classification is that US studies are more numerous (and, hence, deserve a particular focus) and sometimes consider several demographic groups simultaneously (e.g., Pencavel 2002, Devereux 2003). As mentioned before, our focus is on steady-state elasticities, i.e., elasticities from static (structural) models. We separately report uncompensated wage elasticities (total hour and participation responses) and income elasticities. ${ }^{9}$ The uncompensated (Marshallian) wage elasticity is defined as the percentage change in labor supply $h$ for a $1 \%$ change in the (gross) wage $w::^{10}$

$$
\epsilon^{u}=\frac{d h}{d w} \frac{w}{h} .
$$

The income elasticity is defined as the percentage change in labor supply $h$ for a $1 \%$ change in the non-labor labor income $y$ :

$$
\epsilon^{Y}=\frac{d h}{d y} \frac{y}{h} \text {. }
$$

Using the Slutsky equation, it is straightforward to derive the compensated (Hicksian) elasticity (capturing only the substitution effect) as

$$
\epsilon^{c}=\epsilon^{u}-\frac{w h}{y} \epsilon^{Y} \text {. }
$$

The tables highlight methodological differences across studies and notably where elasticities stem from the estimation of continuous labor supply functions (the Hausman approach), from the estimation of discrete-choice models, and from grouped estimations or natural experiments. We can observe an over-representation of studies based on discrete-choice models with taxation, as this method is increasingly used around the world to analyze the effect of fiscal and social policy reforms. ${ }^{11} \mathrm{We}$ do not pretend to be fully exhaustive but nonetheless attempt to give a sense of the range of elasticities obtained in the vast literature for Europe and the USA. Some studies do not report elasticities and unfortunately could not be included in our tables. ${ }^{12}$ This is the case with some studies using labor supply models (e.g., Hoynes 1996 reports income elasticities but not wage elasticities) and more generally the case with studies using policy reforms as natural experiments, as indicated above (for instance, Bingley and Walker 1997, for the UK, or Eissa and Liebman 1996, for the USA). In addition to Tables 1, 2, and 3, the analysis below is supported by graphics obtained using wage-elasticity estimates drawn from these tables (Figs. 1 and 2).

\subsection{Overview}

Figure 1 plots the distribution of wage-elasticity estimates by demographic group. The vertical axis reports the frequency (number of estimates). The first observation is that married women are the group with the largest number of available estimates. The second lesson from these graphs is that, in line with conventional wisdom, elasticities are largest among married women and single mothers, with mean values of .43 and .59 , respectively. These groups also show much dispersion across available studies. While a majority of estimates for married women are found between 0 and .50, estimates for single mothers are far less numerous and more dispersed over a broad range of values. Married and single 
Table 1 Labor supply elasticities in Europe: couples

\begin{tabular}{|c|c|c|c|c|c|c|c|c|c|c|c|}
\hline \multirow[b]{2}{*}{ Country } & \multirow[b]{2}{*}{ Authors } & \multirow[b]{2}{*}{ Data selection } & \multirow[b]{2}{*}{ Model } & \multirow[b]{2}{*}{ Specification } & \multirow[b]{2}{*}{ Tax-benefit } & \multicolumn{2}{|c|}{ Female wage elast. } & \multicolumn{2}{|c|}{ Male wage elast. } & \multicolumn{2}{|c|}{ Income elast. } \\
\hline & & & & & & Hours & Particip. & Hours & Particip. & Female & Male \\
\hline Austria & Dearing et al. (2007) & $\begin{array}{l}\text { SILC (2004), at least } 1 \text { child } \\
\text { aged }<10\end{array}$ & $\mathrm{D}$ & QU;M & ITABENA & & {$[.07, .19]^{\mathrm{b}}$} & & & & \\
\hline \multirow[t]{2}{*}{ Belgium } & Orsini $(2007,2012)$ & $\begin{array}{l}\text { Panel Survey of Belgian } \\
\text { Households (2000-2001), } \\
\text { working age }\end{array}$ & $\mathrm{D}$ & $\begin{array}{l}\text { QU and GU + } \\
\text { PTD; J }\end{array}$ & MODETE & {$[.16, .31]$} & {$[.10, .19]$} & {$[.10, .18]$} & {$[.08, .15]$} & & \\
\hline & Dagsvik et al. (2011) & $\begin{array}{l}\text { National Register Data } \\
\text { (2002), working age }\end{array}$ & $\mathrm{D}$ & Polynomial & MIMOSI & .44 & .21 & .31 & .18 & & \\
\hline \multirow[t]{2}{*}{ Denmark } & Smith (1995) & $\begin{array}{l}\text { Administrative register data } \\
(1980-1986)\end{array}$ & C & $S L$ & $P L$ & .061 & & .093 & & 0 & -.025 \\
\hline & Frederiksen et al. (2008) & $\begin{array}{l}\text { Survey by Statistics Denmark } \\
\text { (1996), age 18-59 }\end{array}$ & C & $S L, F C$ & $P L$ & .148 & & .05 & & -.007 & -.006 \\
\hline \multirow[t]{2}{*}{ Finland } & Kuismainen (1997) & $\begin{array}{l}\text { LFS }(1989) \text {, survey and tax } \\
\text { register; } 25-60\end{array}$ & C & $S L, R$ & $P L$ & {$[0, .06]$} & & & & {$[.11, .27]$} & \\
\hline & Bargain and Orsini (2006) & $\begin{array}{l}\text { IDS (1998), working age, } \\
\text { men all employed }\end{array}$ & $\mathrm{D}$ & $\mathrm{QU}+\mathrm{FC} ; \mathrm{M}$ & EUROMOD & {$[.10, .18]$} & {$[.10, .17]^{\mathrm{a}}$} & & & & \\
\hline \multirow[t]{5}{*}{ France } & $\begin{array}{l}\text { Bourguignon and Magnac } \\
\text { (1990) }\end{array}$ & $\begin{array}{l}\text { LFS (1985), couples aged } \\
18-60\end{array}$ & $\mathrm{C} / \mathrm{T}$ & $L L+R ; M$ or J & $P L, D$ & 1 (.05 with FC) & & .10 & & $\begin{array}{l}-.03(-.02 \\
\text { with } \\
\text { FC)/-.11 a }\end{array}$ & -.07 \\
\hline & Laroque and Salanie (2002) & $\begin{array}{l}\text { Matched LFS-tax returns } \\
\text { (1999), women aged 25-49 }\end{array}$ & $\mathrm{D}$ & $\begin{array}{l}\text { Joint particip. and } \\
\text { wage; unempl. } \\
\text { and min. wage }\end{array}$ & Own calc. & & $(.96)$ & & & & \\
\hline & Choné et al. (2003) & $\begin{array}{l}\text { Matched LFS-tax returns } \\
\text { (1997), working age, children } \\
\text { aged <6 }\end{array}$ & $\mathrm{D}$ & $\begin{array}{l}\text { QU, joint wage } \\
\text { and CC; min. } \\
\text { wage }\end{array}$ & Own calc. & 1.05 & {$[.8, .9]^{\mathrm{b}}$} & & & $-.19 /-.18^{a}$ & \\
\hline & Bargain and Orsini (2006) & $\begin{array}{l}\text { HBS }(1994 / 5) \text {, working age } \\
\text { women, men all employed }\end{array}$ & $\mathrm{D}$ & $\mathrm{QU}+\mathrm{FC} ; \mathrm{M}$ & EUROMOD & {$[.52, .65]$} & {$[.46, .58]^{\mathrm{a}}$} & & & & \\
\hline & Donni and Moreau (2007) & $\begin{array}{l}\text { HBS (2001), aged 20-60, } \\
\text { all employed, no children } \\
\text { aged }<3\end{array}$ & C & $\begin{array}{l}\text { QL; s-conditional } \\
\text { collective LS }\end{array}$ & No taxation & {$[.24,59]$} & & & & {$[-.35,-.06]$} & \\
\hline
\end{tabular}


Table 1 Labor supply elasticities in Europe: couples (Continued)

\begin{tabular}{|c|c|c|c|c|c|c|c|c|c|c|c|}
\hline \multirow[t]{11}{*}{ Germany } & Kaiser et al. (1992) & SOEP (1983), working age & C & $\mathrm{LL}$ & $C, N C, D$ & 1.04 & & -.04 & & -.18 & -.28 \\
\hline & Bonin et al. (2002) & $\begin{array}{l}\text { SOEP (2000), working age, W } \\
\text { and E }\end{array}$ & $\mathrm{D}$ & TL + PTD; J & IZAmod & .27 & .20 & .21 & .19 & $.15 / .09$ & $.01 / 0$ \\
\hline & Steiner and Wrohlich (2004) & $\begin{array}{l}\text { SOEP (2002), working age, W } \\
\text { and E }\end{array}$ & D & TU + PTD; J & STSM & {$[.16, .55]^{\mathrm{b}}$} & {$[.07, .21]^{\mathrm{b}}$} & {$[.11, .38]^{\mathrm{b}}$} & {$[.07, .23]^{b}$} & & \\
\hline & Haan and Steiner (2004) & $\begin{array}{l}\text { SOEP (2002), working age, W } \\
\text { and E, one- or two-earner cou- } \\
\text { ples }\end{array}$ & $\mathrm{D}$ & TU + PTD; J & STSM & {$[.08, .56]$} & {$[.04,20]$} & {$[.08, .46]$} & {$[.07, .26]$} & & \\
\hline & Bargain and Orsini (2006) & $\begin{array}{l}\text { SOEP (1998), working age, men } \\
\text { all employed, W and E }\end{array}$ & D & $\mathrm{QU}+\mathrm{FC} ; \mathrm{M}$ & EUROMOD & {$[.31, .45]$} & {$[.27, .38]^{a}$} & & & & \\
\hline & Haan (2006) & $\begin{array}{l}\text { SOEP }(2001), W \text { and E; married } \\
\text { couples, } 20-65 \text { years }\end{array}$ & $\mathrm{D}$ & TU & STSM & {$[.34, .39]$} & {$[.13, .14]$} & {$[.19, .22]$} & {$[.12, .14]$} & & \\
\hline & Clauss and Schnabel (2006) & $\begin{array}{l}\text { SOEP }(2004 / 2005), \text { couples } \\
\text { aged 20-65 }\end{array}$ & $D$ & $\mathrm{TU} ; \mathrm{J}$ & STSM & .37 & .14 & .24 & .16 & & \\
\hline & Wrohlich (2006) & $\begin{array}{l}\text { SOEP (2002), working age, W } \\
\text { and E }\end{array}$ & D & $\mathrm{TU} ; \mathrm{J} ; \mathrm{CC}$ & STSM & {$[.14, .53]^{\mathrm{b}}$} & {$[.06, .16]^{\mathrm{b}}$} & & & & \\
\hline & Dearing et al. (2007) & $\begin{array}{l}\text { SOEP }(2004) \text {, at least } 1 \text { child } \\
\text { aged }<10, \mathrm{~W}\end{array}$ & D & QU; M & STSM & & {$[.13, .24]^{b}$} & & & & \\
\hline & Bargain et al. (2010) & $\begin{array}{l}\text { SOEP (2003), working age, } \\
\text { potential one- or two-earner }\end{array}$ & $\mathrm{D} / \mathrm{H}$ & $\mathrm{QU}+\mathrm{PTD}, \mathrm{R} ; \mathrm{J}$ & STSM & {$[.19, .34]$} & {$[.08,20]$} & {$[.05, .08]$} & {$[.04, .13]$} & & \\
\hline & Fuest et al. (2008) & $\begin{array}{l}\text { SOEP (2004), working age, W } \\
\text { and E, potential one- or } \\
\text { two-earner }\end{array}$ & $\mathrm{D}$ & TU+PTD;J & FiFoSiM & .38 & .15 & .20 & .14 & & \\
\hline \multirow[t]{2}{*}{ Ireland } & Callan and van Soest (1996) & IDS (1987), desired hours & $\mathrm{D} / \mathrm{H}$ & $T U+F C, R$; J & SWITCH & {$[.50, .85]$} & $.31 / .20^{\mathrm{a}}$ & {$[.10, .20]$} & & & \\
\hline & Callan et al. (2009) & $\begin{array}{l}\text { Living in Ireland Survey (1995), } \\
\text { desired hours }\end{array}$ & D & $T U+F C, R ; J$ & SWITCH & {$[.71, .90]$} & .49 & {$[.21, .31]$} & $.20 / .21^{\mathrm{a}}$ & & \\
\hline \multirow[t]{2}{*}{ Italy } & Colombino and del Boca (1990) & $\begin{array}{l}\text { Turin Survey of Couples (1979), } \\
\text { working age }\end{array}$ & C & LL & $P L$ & 1.18 & .64 & & & .52 & \\
\hline & Aaberge et al. (1999) & $\begin{array}{l}\text { Survey of Income and Wealth } \\
\text { (1987), aged 20-70 }\end{array}$ & A & $\begin{array}{l}\text { Non-linear hours, } \\
\text { exog. wage and } \\
\text { unearned inc. }\end{array}$ & Own calc. & .74 & .65 & .053 & .046 & -.014 & -.003 \\
\hline
\end{tabular}


Table 1 Labor supply elasticities in Europe: couples (Continued)

\begin{tabular}{|c|c|c|c|c|c|c|c|c|c|c|}
\hline & $\begin{array}{l}\text { Aaberge et al. } \\
(2002,2004)\end{array}$ & $\begin{array}{l}\text { Survey of Income and Wealth } \\
\text { (1993) }\end{array}$ & A & GU; J & Own calc. & .66 & .51 & .12 & .02 & \\
\hline \multirow[t]{7}{*}{ Netherlands } & $\begin{array}{l}\text { van Soest et al. } \\
\text { (1990) }\end{array}$ & $\begin{array}{l}\text { Labor mobility survey (1985), } \\
\text { working age }\end{array}$ & $C / D$ & $\begin{array}{l}\mathrm{LL}, \quad \mathrm{R} \text {; discrete } \\
\text { wage-hours } \\
\text { combinations }\end{array}$ & $P L$ & {$[.35, .59]$} & .12 & {$[.15, .19]$} & -.23 & -.01 \\
\hline & van Soest (1995) & SOEP (1987) & D & $T U$ + PTD, R; J & Own calc. & {$[.42, .54]$} & & {$[.05, .09]$} & .008 & -.03 \\
\hline & $\begin{array}{l}\text { van Soest and Das } \\
(2001)\end{array}$ & $\begin{array}{l}\text { SOEP (1995), aged 16-64, } \\
\text { desired hours }\end{array}$ & D & $T U+F C, R ; J$ & Own calc. & {$[.67, .74]$} & & {$[.07, .10]$} & & \\
\hline & $\begin{array}{l}\text { van Soest et al. } \\
(2002)\end{array}$ & $\begin{array}{l}\text { Dutch SOEP (1995), aged 16-64, } \\
\text { desired hours }\end{array}$ & D & $\begin{array}{l}\text { QU (+ more } \\
\text { flexible) }+ \text { FC, } \\
\text { R; simult. wage } \\
\text { estimation, J }\end{array}$ & Own calc. & {$[.83,1.36]$} & {$[.35, .58]^{a}$} & & & \\
\hline & Bloemen (2009) & $\begin{array}{l}\text { SEP (1990-2001), couples w/o } \\
\text { children, age 22-60 }\end{array}$ & D & QL & Own & {$[.22,61]$} & & {$[.24,61]$} & -.057 & \\
\hline & Bloemen (2010) & $\begin{array}{l}\text { SEP (1990-2002), couples w/o } \\
\text { children, age 22-60 }\end{array}$ & D & QU, FC & Own & {$[.14, .31]$} & & {$[-.02, .03]$} & & \\
\hline & $\begin{array}{l}\text { Mastrogiacomo } \\
\text { et al. (2013) }\end{array}$ & $\begin{array}{l}\text { Labour Market Panel } \\
\text { (1999-2005) }\end{array}$ & D & QU, FC & CPB model & {$[.22, .52]$} & {$[.17, .40]$} & {$[.05, .19]$} & {$[.05, .16]$} & \\
\hline \multirow[t]{2}{*}{ Norway } & $\begin{array}{l}\text { Dagsvik and Strøm } \\
(2006)\end{array}$ & $\begin{array}{l}\text { Survey of Income and Wealth } \\
\text { (1994/1995); married couples }\end{array}$ & D & Polynomial & Statistic Norway model & .65 & .28 & & & \\
\hline & $\begin{array}{l}\text { Aaberge and } \\
\text { Colombino (2012) }\end{array}$ & $\begin{array}{l}\text { Survey of Income and Wealth } \\
\text { (1994/1995); married couples }\end{array}$ & D & Polynomial & Statistic Norway model & .21 & .31 & .23 & .16 & \\
\hline \multirow[t]{4}{*}{ Spain } & $\begin{array}{l}\text { García and Suárez } \\
\text { (2003) }\end{array}$ & $\begin{array}{l}\text { ECHP (1994-1995), aged 16-65, } \\
\text { obs. and desired hours }\end{array}$ & C & $\mathrm{LL}$ & Taxes & .37 & $1.51^{\mathrm{a}}$ & & -.06 & \\
\hline & $\begin{array}{l}\text { Fernández-Val } \\
\text { (2003) }\end{array}$ & $\begin{array}{l}\text { ECHP (1994-1999), aged <65 } \\
\text { and in work }\end{array}$ & C & $\begin{array}{l}\text { Unitary/collective } \\
\text { model }\end{array}$ & No taxation & .31 & & & & \\
\hline & Crespo (2006) & $\begin{array}{l}\text { ECHP (1994-1999), aged <65 } \\
\text { and in work }\end{array}$ & C & $\begin{array}{l}\mathrm{QL}, \\
\text { unitary/collective }\end{array}$ & No taxation & .14 & & .01 & & \\
\hline & $\begin{array}{l}\text { Labeaga et al. } \\
(2008)\end{array}$ & ECHP (1995), working age & D & $\mathrm{QU}+\mathrm{FC}_{i} J$ & GLAD- HISPANIA & .29 & .26 & .01 & .11 & \\
\hline
\end{tabular}


Table 1 Labor supply elasticities in Europe: couples Continued

\begin{tabular}{|c|c|c|c|c|c|c|c|c|c|c|c|}
\hline \multirow[t]{6}{*}{ Sweden } & \multirow{2}{*}{$\begin{array}{l}\text { Blomquist (1983) } \\
\text { Flood and MaCurdy } \\
\text { (1992) }\end{array}$} & \multirow{2}{*}{$\begin{array}{l}\text { Level of Living Survey (1974), all } \\
\text { employed, aged 25-55 } \\
\text { Household Market-Nonmarket } \\
\text { Survey (1983), all employed, } \\
25-65\end{array}$} & \multirow{2}{*}{ C } & \multirow{2}{*}{$\begin{array}{l}\mathrm{LL}, \mathrm{R} \\
\mathrm{LL} \text { and } \mathrm{SL}, \mathrm{R}\end{array}$} & \multicolumn{3}{|l|}{$\mathrm{PL}$} & \multicolumn{3}{|l|}{.008} & \multirow{2}{*}{$\begin{array}{l}-.03 \\
{[-.01, .04]}\end{array}$} \\
\hline & & & & & $P L, D$ & & & \multicolumn{3}{|l|}{$[-.25, .21]$} & \\
\hline & $\begin{array}{l}\text { Blomquist and } \\
\text { Hansson-Brusewitz } \\
\text { (1990) }\end{array}$ & $\begin{array}{l}\text { Level of Living Survey (1981), all } \\
\text { employed, aged 25-55 }\end{array}$ & C & $\mathrm{LL}$ and $\mathrm{QL}, \mathrm{R}$ & $\mathrm{PL}, \mathrm{C}$ and $\mathrm{NC}$ & \multicolumn{2}{|l|}{$[.38, .77]$} & \multicolumn{2}{|l|}{$[.08, .13]$} & {$[-.24,-.03]$} & \\
\hline & $\begin{array}{l}\text { Blomquist and Newey } \\
\text { (2002) }\end{array}$ & $\begin{array}{l}\text { Level of Living Survey (1973, } \\
\text { 1980, 1990), all employed, aged } \\
\text { 18-60 }\end{array}$ & C & $\begin{array}{l}\text { Non-parametric } \\
\text { labor supply }\end{array}$ & $P L$ & & & \multicolumn{3}{|l|}{$[.04, .12$} & \\
\hline & Flood et al. (2004) & $\begin{array}{l}\text { Household Income Survey } \\
\text { (1993), aged 18-64 }\end{array}$ & $\mathrm{D}$ & TU, R; stigma of W & Own calc. & \multicolumn{2}{|l|}{.12} & 0 & & \multirow[t]{2}{*}{-.017} & \multirow[t]{2}{*}{-.003} \\
\hline & Brink et al. (2007) & $\begin{array}{l}\text { Longitudinal Individual Data, } \\
\text { Income Distribution Survey, } \\
1999\end{array}$ & $\mathrm{D}$ & $T U, R$ & FASIT & .18 & .15 & .06 & 0 & & \\
\hline Switzerland & Gerfin and Leu (2003) & $\begin{array}{l}\text { Swiss Income and Expenditure } \\
\text { Survey (1998) }\end{array}$ & $\mathrm{D}$ & $\begin{array}{l}\text { Quadratic util- } \\
\text { ity, random } \\
\text { preferences }\end{array}$ & Tax model for Basel-Stadt & .56 & .36 & .03 & .01 & $-.06 /-.04$ & $-.001 / 0$ \\
\hline \multirow[t]{3}{*}{ UK } & $\begin{array}{l}\text { Arellano and Meghir } \\
\text { (1992) }\end{array}$ & $\begin{array}{l}\text { British FES and LFS (1983), aged } \\
20-59 \text {, with pre-school } \\
\text { children (upper bound for all } \\
\text { children) }\end{array}$ & C & $\begin{array}{l}\mathrm{SL}+\mathrm{FC} \text {, search } \\
\text { costs, endoge- } \\
\text { nous wage and } \\
\text { unearned income } \\
\text { (IV) }\end{array}$ & $P L$ & {$[.29, .71]$} & - & & & {$[-.13,-.40]$} & \\
\hline & $\begin{array}{l}\text { Arrufat and Zabalza } \\
\text { (1986) }\end{array}$ & $\begin{array}{l}\text { British General Household } \\
\text { Survey (1974), aged <60 }\end{array}$ & C & $\begin{array}{l}\text { CES utility-based } \\
\text { labor supply, R }\end{array}$ & $P L$ & \multirow[t]{2}{*}[.62-2.03]{} & \multirow[t]{2}{*}{1.41} & & & $-.2 /-.14$ & \\
\hline & $\begin{array}{l}\text { Blundell and Walker } \\
\text { (1986) }\end{array}$ & $\begin{array}{l}\text { FES (1980), all employed, aged } \\
\text { 18-59 }\end{array}$ & C & $\begin{array}{l}\text { Gorman polar } \\
\text { form and translog } \\
\text { hours, R }\end{array}$ & $P L$ & & & \multicolumn{3}{|l|}{.024} & -.287 \\
\hline
\end{tabular}


Table 1 Labor supply elasticities in Europe: couples (Continued)

\begin{tabular}{|c|c|c|c|c|c|c|c|c|}
\hline Blundell et al. (1987) & FES (1981), aged 16- & & $T / H$ & $\begin{array}{l}\text { Non-linear } \\
\text { labor supply, } \\
\text { unemployment } \\
\text { risk }\end{array}$ & Own calc. & & {$[.0, .408]$} & \\
\hline Blundell et al. (1998) & $\begin{array}{l}\text { FES (1978-1992), } \\
\text { young children } \\
\text { bound if no child) }\end{array}$ & $\begin{array}{l}\text { 20-50, } \\
\text { (lower }\end{array}$ & C & $\begin{array}{l}\text { Generalized LES, } \\
\mathrm{R}\end{array}$ & $P L$ & {$[.13, .37]^{b}$} & - & {$[-.19,0]^{b}$} \\
\hline Blundell et al. (2000) & $\begin{array}{l}\text { Family Resources } \\
(1994-1996)\end{array}$ & Survey & $\mathrm{D}$ & $\mathrm{QU}+\mathrm{FC}, \mathrm{R}, \mathrm{W}$ & TAXBEN & {$[.11-.17]$} & & \\
\hline
\end{tabular}


Table 2 Labor supply elasticities in Europe: single individuals

\begin{tabular}{|c|c|c|c|c|c|c|c|c|}
\hline \multirow[b]{2}{*}{ Country } & \multirow[b]{2}{*}{ Authors } & \multirow[b]{2}{*}{ Data selection } & \multirow[b]{2}{*}{ Model } & \multirow[b]{2}{*}{ Specification } & \multirow[b]{2}{*}{ Tax-benefit } & \multicolumn{2}{|c|}{ Wage elast. } & \multirow{2}{*}{$\begin{array}{c}\text { Income } \\
\text { Elast. }\end{array}$} \\
\hline & & & & & & Hours & Particip. & \\
\hline \multirow[t]{2}{*}{ Belgium } & Dagsvik et al. (2011) & $\begin{array}{l}\text { National Register Data, 2002, } \\
\text { working age, SW }\end{array}$ & $\mathrm{D}$ & Polynomial & MIMOSI & .13 & .07 & \\
\hline & & SM & & & & .2 & .11 & \\
\hline Finland & Bargain and Orsini (2006) & IDS (1998), SW, SP & $\mathrm{D}$ & $\mathrm{QU}+\mathrm{FC}$ & EUROMOD & {$[.18, .34]$} & {$[.18, .33]$} & \\
\hline \multirow[t]{2}{*}{ France } & Bargain and Orsini (2006) & $\begin{array}{l}\text { HBS (1994/1995), aged 25-49, } \\
\text { SW, SP }\end{array}$ & $\mathrm{D}$ & $\mathrm{QU}+\mathrm{FC}$ & EUROMOD & {$[.08, .14]$} & {$[.04, .07]$} & \\
\hline & Laroque and Salanie (2002) & $\begin{array}{l}\text { LFS-tax return matched dataset } \\
\text { (1999), women aged 25-49, no } \\
\text { civil servants, SW }\end{array}$ & D & $\begin{array}{l}\text { Participation (and full/part-time) } \\
\text { model, simultaneous wage and } \\
\text { labor supply estimation, probability } \\
\text { of unemployment, min. wage }\end{array}$ & Own calc. & & .36 & \\
\hline \multirow[t]{11}{*}{ Germany } & Bargain and Orsini (2006) & SOEP (1998), SW, SP & $\mathrm{D}$ & $\mathrm{QU}+\mathrm{FC}$ & EUROMOD & {$[.09, .18]$} & {$[.08, .15]$} & \\
\hline & Steiner and Wrohlich (2004) & SOEP (2003), SW & $\mathrm{D}$ & TU + PTD & STSM & {$[.20, .36]$} & {$[.05, .09]$} & \\
\hline & Haan and Steiner (2004) & SOEP (2002), SW & D & TU + PTD & STSM & {$[.02, .24]$} & {$[.01, .10]$} & \\
\hline & & SM & & & & {$[.08, .31]$} & {$[.04, .28]$} & \\
\hline & Clauss and Schnabel (2006) & $\begin{array}{l}\text { SOEP (2004/2005), aged 20-65, } \\
\text { SW }\end{array}$ & $\mathrm{D}$ & TU + PTD & STSM & .38 & .18 & \\
\hline & & SM & & & & .23 & .17 & \\
\hline & Haan and Uhlendorff (2007) & $\begin{array}{l}\text { SOEP (2000-2005), age 25-59, } \\
\text { SM }\end{array}$ & $\mathrm{D}$ & $\begin{array}{l}\text { Reduced form risk model; } \\
\text { non-parametric random } \\
\text { coefficient }\end{array}$ & STSM & {$[.016, .036]$} & {$[.05, .12]$} & \\
\hline & Fuest et al. (2008) & SOEP (2004), working age, SW & $\mathrm{D}$ & $T U+P T D$ & FiFoSiM & .28 & .13 & \\
\hline & & SM & & & & .28 & .17 & \\
\hline & Bargain et al. (2010) & SOEP (2003), working age, SW & $\mathrm{D} / \mathrm{H}$ & $\begin{array}{l}\text { QU + PTD; involuntary } \\
\text { unemployment }\end{array}$ & STSM & {$[.06, .16]$} & {$[.04, .10]$} & \\
\hline & & SM & & & & {$[.10, .20]$} & {$[.05, .12]$} & \\
\hline \multirow[t]{2}{*}{ Italy } & Aaberge et al. (2002) & $\begin{array}{l}\text { Survey on Household Income } \\
\text { and Wealth (1993), SW }\end{array}$ & A & GU & Own calc. & .10 & .06 & \\
\hline & & SM & & & & .11 & .08 & \\
\hline
\end{tabular}


Table 2 Labor supply elasticities in Europe: single individuals (Continued)

\begin{tabular}{|c|c|c|c|c|c|c|c|c|}
\hline \multirow[t]{4}{*}{ Netherlands } & \multirow[t]{2}{*}{ Euwals and Van Soest (1999) } & $\begin{array}{l}\text { Dutch SOEP (1988), actual } \\
\text { and desired hours, SW }\end{array}$ & \multirow[t]{2}{*}{ D } & \multirow[t]{2}{*}{$T U+F C, R$} & \multirow[t]{2}{*}{ Own calc. } & \multicolumn{3}{|l|}{$[.03, .45]$} \\
\hline & & SM & & & & \multicolumn{3}{|l|}{$[.03, .18]$} \\
\hline & \multirow[t]{2}{*}{ Mastrogiacomo et al. (2013) } & $\begin{array}{l}\text { Labour Market Panel, } \\
\text { 1999-2005, SW }\end{array}$ & \multirow[t]{2}{*}{ D } & \multirow[t]{2}{*}{$\mathrm{QU}, \mathrm{FC}$} & \multirow[t]{2}{*}{ CPB Model } & {$[.04, .62]$} & {$[.01, .43]$} & \\
\hline & & SM & & & & {$[.14, .45]$} & {$[.09, .32]$} & \\
\hline \multirow[t]{2}{*}{ Norway } & \multirow[t]{2}{*}{ Aaberge and Colombino (2012) } & $\begin{array}{l}\text { Survey of Income and } \\
\text { Wealth (1994/1995); SW }\end{array}$ & \multirow[t]{2}{*}{ D } & \multirow[t]{2}{*}{ Polynomial } & \multirow[t]{2}{*}{ Statistic Norway model } & -.09 & .12 & \\
\hline & & SM & & & & -.02 & .04 & \\
\hline \multirow[t]{2}{*}{ Sweden } & Andrén (2003) & HINK (1997-1998), SP & $\mathrm{D}$ & $\begin{array}{l}\mathrm{QU}+\mathrm{FC} \text {; simulat. with W and } \\
\mathrm{CC}\end{array}$ & Own calc. & {$[.55, .87]$} & .50 & -.1 \\
\hline & Brink et al. (2007) & $\begin{array}{l}\text { Longitudinal Individual Data, } \\
\text { IDS, 1999, SP }\end{array}$ & $\mathrm{D}$ & $T U, R$ & FASIT & .51 & .35 & \\
\hline \multirow[t]{5}{*}{ UK } & Walker (1990) & FES (1979-1984), SP & $\mathrm{D}$ & Participation model & \multicolumn{2}{|l|}{ Benefits only } & .70 & \\
\hline & Ermisch and Wright (1991) & $\begin{array}{l}\text { General household survey } \\
\text { (1973-1982), SP }\end{array}$ & D & $\begin{array}{l}\text { Participation model, } \\
\text { demand-side controls }\end{array}$ & \multicolumn{2}{|l|}{ Simplified system } & 1.7 & \\
\hline & Jenkins (1992) & $\begin{array}{l}\text { Lone parents survey (1989), } \\
\text { SP }\end{array}$ & $\mathrm{D}+\mathrm{H}$ & $\begin{array}{l}\text { Two positive hour choices, } \\
\text { unemployment risk, FC }\end{array}$ & \multicolumn{2}{|l|}{ Benefits only } & 1.8 & \\
\hline & Blundell et al. (1992) & FES (1981-1986), SP & C & $\begin{array}{l}\text { Marginal rate of substitution } \\
\text { function, endogenous wage } \\
\text { and income }\end{array}$ & \multicolumn{2}{|l|}{ Taxation only } & .34 & \\
\hline & Brewer et al. (2006) & $\begin{array}{l}\text { FES (1995-2002), aged <60, } \\
\text { SP }\end{array}$ & $\mathrm{D}$ & $\begin{array}{l}\mathrm{QU}+\mathrm{FC} \text {, joint with } \mathrm{W} \text { and } \\
\mathrm{CC}, \mathrm{R}\end{array}$ & \multicolumn{2}{|l|}{ TAXBEN } & 1.02 & \\
\hline \multicolumn{9}{|c|}{ 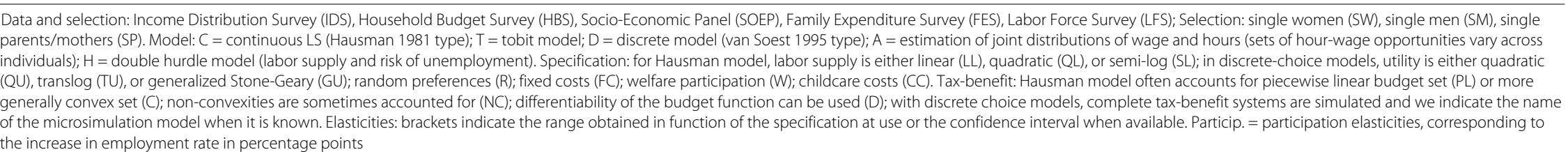 } \\
\hline
\end{tabular}


Table 3 Labor supply elasticities for the USA

\begin{tabular}{|c|c|c|c|c|c|c|c|c|c|}
\hline \multirow[b]{2}{*}{ Authors } & \multirow[b]{2}{*}{ Data selection } & \multirow[b]{2}{*}{ Model } & \multirow[b]{2}{*}{ Specification } & \multicolumn{2}{|c|}{ Female wage elast. } & \multicolumn{2}{|c|}{ Male wage elast. } & \multicolumn{2}{|c|}{ Income elast. } \\
\hline & & & & Hours & Particip. & Hours & Particip. & Female & Male \\
\hline Cogan (1981) & $\begin{array}{l}\text { US NLSW 1967, married women aged } \\
\text { 30-35 }\end{array}$ & C & $\begin{array}{l}\text { SL; reservation hours to } \\
\text { account for } F C \text {; no tax-benefit }\end{array}$ & {$[.86,2.40]$} & & & & {$[.16, .66]$} & \\
\hline Hausman (1981) & PSID 1975, married women & C & $\mathrm{LL}, \mathrm{PL}(\mathrm{C}$ and NC: FC) & {$[.90,1.00]$} & & & & {$[-.13,-.12]$} & \\
\hline Triest (1990) & PSID 1983, married women, aged 25-55 & C & $\mathrm{LL} ; \mathrm{C}$ and $\mathrm{PL} ;$ taxes and benefits & {$[.03, .28]$} & & & & {$[-.15,-.19]$} & \\
\hline MaCurdy et al. (1990) & PSID 1975: married men, aged 25-55 & C & $\begin{array}{l}\mathrm{LL} ; \mathrm{PL} \text { and } \mathrm{D} \text { (reconvexified) } \\
\text { budget set; taxes }\end{array}$ & & & {$[-.24, .03]$} & & -.01 & \\
\hline Dickert et al. (1995) & SIPP 1990, single mothers, no assets & D & $\begin{array}{l}\text { Joint program and labor force } \\
\text { participation }\end{array}$ & & .35 & & & & \\
\hline \multirow[t]{2}{*}{ Pencavel (1998) } & $\begin{array}{l}\text { CPS 1975-1994, married women, aged } \\
\text { 25-60 }\end{array}$ & C & Log-L; no tax-benefit & & {$[.77, .1 .80]$} & & & & \\
\hline & $\begin{array}{l}\text { CPS 1975-1994, single women, aged } \\
25-60\end{array}$ & & & {$[.77, .1 .80]$} & & & & & \\
\hline Hoynes (1996) & $\begin{array}{l}\text { SIPP panel, 1984, married men and } \\
\text { women with children }\end{array}$ & D & $\begin{array}{l}\text { Stone-Geary; stigma from } \\
\text { AFDC; tax-benefit system; FC }\end{array}$ & & & & & -.46 & -.12 \\
\hline Keane and Moffitt (1998) & 1994 SIPP, single mothers, no assets & D & $\begin{array}{l}\text { Joint labor supply and welfare } \\
\text { program participation; benefits } \\
\text { but no tax }\end{array}$ & & .96 & & & & \\
\hline \multirow[t]{2}{*}{ Pencavel (2002) } & CPS 1999, married men & C & LL; no tax-benefit & & & {$[.12, .25]$} & & & \\
\hline & CPS 1999, single men & & & & & {$[.12, .25]$} & & & \\
\hline \multirow[t]{2}{*}{ Devereux (2003) } & Census and PSID, married men & C & Log-L, no tax-benefit & & & {$[-.022, .017]$} & {$[-.061, .001]$} & & \\
\hline & Single men & & & & & {$[-.022, .017]$} & {$[-.061, .001]$} & & \\
\hline Devereux (2004) & $\begin{array}{l}\text { PUMS 1980, 1990, married couples } \\
\text { (participating men) }\end{array}$ & C & Log-L, no tax-benefit & {$[.17,38]$} & & {$[.00, .07]$} & & & \\
\hline Eissa and Hoynes (2004) & $\begin{array}{l}\text { CPS } 1985 \text { to } 1997 \text {, less educated married } \\
\text { couples with children }\end{array}$ & D & $\begin{array}{l}\text { Participation Probit, joint } \\
\text { estimation }\end{array}$ & & .27 & & .03 & -.039 & -.007 \\
\hline \multirow[t]{3}{*}{ Blau and Kahn (2007) } & $\begin{array}{l}\text { CPS 1980, married men and women age } \\
25-54\end{array}$ & C & Log-L & {$[.77, .88]$} & & {$[.01, .07]$} & & .004 & .001 \\
\hline & CPS 1990 & C & Log-L & {$[.58,64]$} & & {$[.10, .14]$} & & .002 & .002 \\
\hline & CPS 2000 & C & Log-L & {$[.36, .41]$} & & {$[.04, .10]$} & & .001 & .002 \\
\hline
\end{tabular}


Table 3 Labor supply elasticities for the USA (Continued)

\begin{tabular}{|c|c|c|c|c|c|c|c|c|}
\hline Heim (2007) & $\begin{array}{l}\text { CPS, 1979-2003, married } \\
\text { women }\end{array}$ & $\begin{array}{l}\text { SL, participation, some account } \\
\text { for tax }\end{array}$ & .36 (1979) to .14 (2003) & .66 (1979) to .03 (2003) & & & $-.05(1979)$ to -.015 (2003) & \\
\hline Heim (2009) & PSID 2001, couples & $\begin{array}{l}\text { Quadratic utility with continuous } \\
\text { labor supply, J, FC, R }\end{array}$ & {$[.24,33]$} & {$[.07, .18]$} & {$[.04, .07]$} & {$[.00, .003]$} & {$[-.007,-.006]$} & -.0007 \\
\hline Bishop et al. (2009) & $\begin{array}{l}\text { CPS, 1979-2003, sing. } \\
\text { women }\end{array}$ & $\begin{array}{l}S L \text {, participation, some account } \\
\text { for tax }\end{array}$ & .14 (1979) to -.03 (2003) & .28 (1979) to $22(2003)$ & & & $-.014(1979)$ to -.019 (2003) & \\
\hline
\end{tabular}
Data: Current Population Survey (CPS), National Longitudinal Surveys of Young Women and Mature Women (NLSW), Panel Study on Income Dynamics (PSID), Public Use Microdata Sample (PUMS), Survey of Income and Program
Participation (SIPP). Model: C= continuous labor supply (Hausman 1981 type); D = discrete-choice model (often a simple participation probit). Specification: Hausman labor supply is either linear (LL), log-linear (Log-L), or semi-log (SL); Participation (SIPP). Model: $\mathrm{C}=$ continuous labor supply (Hausman 1981 type); $\mathrm{D}=$ discrete-choice model (often a simple participation probit). Specification: Hausman labor supply is either linear (LL), log-linear (Log-L), or semi-log (SL);
random preferences are sometimes accounted for (R) as well as fixed costs (FC). Models sometimes account for piecewise linear budget set (PL) or more generally convex set (C) or non-convexities (NC) and differentiable budget constraint (D). Elasticities: brackets indicate ranges of values over different specifications or reported confidence intervals. Participation elasticities ("particip"): increase in employ. rate in \% points 

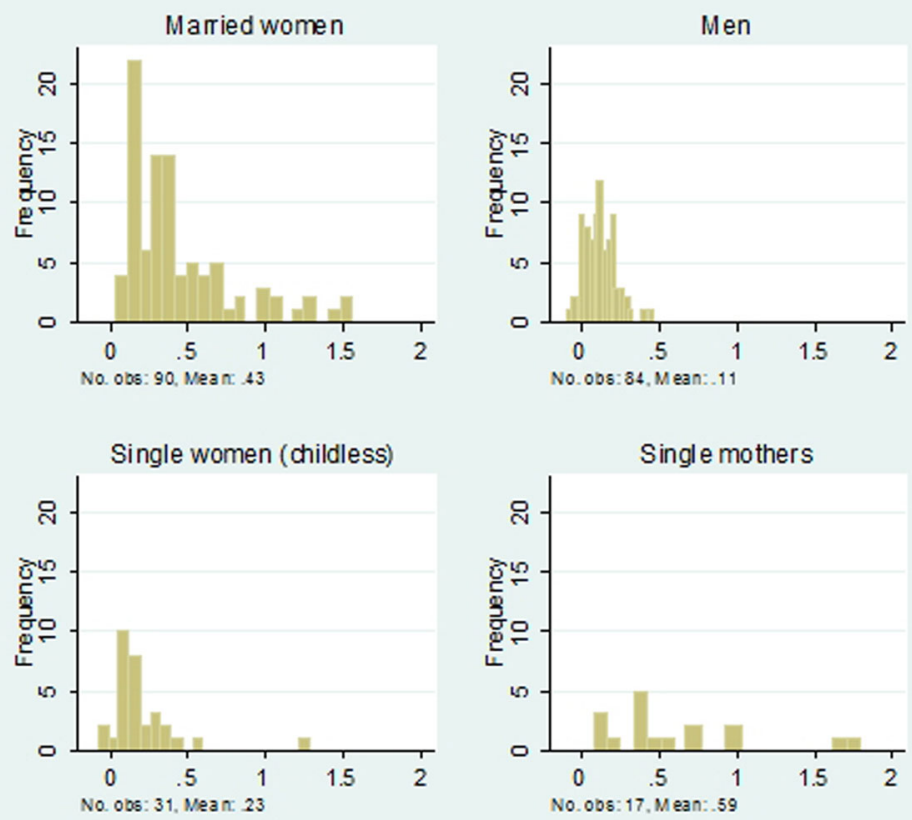

Fig. 1 Distribution of wage elasticities by demographic group
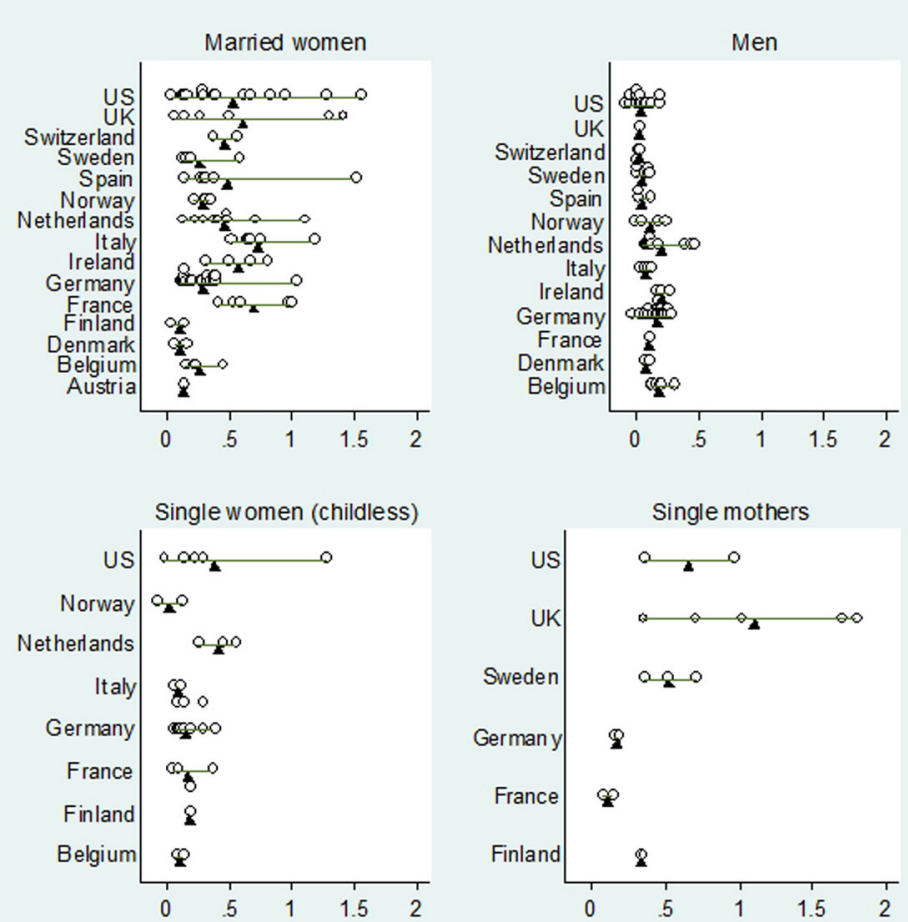

Fig. 2 Distribution of wage elasticities by demographic group and country 
men (mean value .12) and childless single women (mean value .23) show much less variation, and most estimates stand in a narrow range between 0 and .30 . These conclusions do not change radically if we consider more specific types of elasticities, namely total hour elasticities or participation elasticities (detailed results available from the authors). We now discuss each demographic group specifically.

\subsection{Demographic groups}

Married women Considering Tables 1 and 3, we observe much dispersion in estimates for married women. This is confirmed in Fig. 2 (top-left quadrant) where we plot the distribution of wage-elasticity estimates for each country. The black triangular cursors indicate mean values over all available estimates for each country. Mean elasticities for the UK and the USA hide a very broad dispersion across studies. Difference in elasticity size may be driven by heterogeneity in work preferences across countries and over time or by methodological reasons.

As far as genuine international differences are concerned, we suggest that larger wage elasticities prevail in countries where women's participation is low: This seems to be the case in our survey estimates for Ireland and Italy, which is confirmed in the discussions in Callan et al. (2009) and Aaberge et al. (2002) for these two countries, respectively. In contrast, women's participation is high in Nordic countries and participation elasticities tend to be fairly small there, notably in Finland and Denmark and also Sweden and Norway. An exception is Blomquist and Hansson-Brusewitz (1990) for Sweden, but the authors examine data from the 1980s. Comparing Italy and Norway/Sweden, Aaberge et al. (1999) show that lower participation rates among married women in Italy leads to a larger potential for reforms that increase financial incentives to work. Larger elasticities coincide with more intermittent labor force participation patterns in Southern countries and Ireland, as opposed to more consistent participation and more constant hours in Scandinavian countries. Apart from these extreme cases, differences across EU countries, and notably countries of Continental Europe, may not be very large, as suggested by Evers et al. (2008). This is confirmed by Bargain et al. (2014): Using a harmonized framework for $17 \mathrm{EU}$ countries and the USA, for the same time period, they find estimates for married women ranging in a narrow interval .2-.6. This is indeed where mean values lie in Fig. 2 (top-left quadrant), with few exceptions. They also confirm that most of the responses occur at the extensive (participation) margin. Contrary to this study, our meta-analysis addresses comparisons across studies based on different methodological options, notably the period of investigation and the estimation method. We investigate the role of these two factors in the next subsection.

Single mothers This demographic group has received some attention in the literature because of its importance for welfare analysis, given its higher risk of poverty, and because single-parent families were primarily concerned with reforms like tax credit extensions in the USA (cf. Hotz and Scholz 2003) or the UK (Blundell et al. 2000). This group is found to be more responsive to financial incentives than the average, at least in the UK, the USA, and Sweden. This is confirmed in Tables 2 and 3, where relatively large elasticities are shown in several studies, but not all. There is indeed much variance across estimates for lone mothers, in particular for the UK, as can be seen in Fig. 2 (bottom-right quadrant). Moderate estimates are found in some studies for the UK (Blundell et al. 1992) and the 
USA (Dickert et al. 1995) while other papers point to much larger elasticities (e.g., Keane and Moffitt 1998, for the USA or many of the British studies). The largest responses occur at the extensive margin in the UK, with very high participation elasticities on data from the 1990s and also in more recent studies (Brewer et al. 2006).

It is noticeable that the group of single parents has become much larger in the recent period. This is particularly the case in Anglo-Saxon countries, which implies possible changes in the selection effect. That is, this group may be less negatively selected in terms of labor market participation in the recent period. For the USA, Bishop et al. (2009) study all single women over a long period (1979-2003), using a simple estimation of hours and participation on repeated cross sections. They report a significant decline in hour-wage elasticities over the period and relatively small elasticities in the recent years (at least compared to typical estimates for married women).

Men and childless single individuals There is a long history of estimating male labor supply (see surveys of Hausman 1985b and Pencavel 1986, for married men). Estimates of wage elasticities for this group are usually very small, often not significant, and sometimes negative. Studies reported in Table 1 broadly confirm these stylized facts for married men. There are few exceptions, with larger elasticities in Ireland and in some of the German studies. Evidence for childless single men and women, gathered in Table 2, is relatively limited, despite the growing proportion of this demographic group in the population.

This limited evidence is essentially explained by methodological reasons. First, estimates are usually more precise for couples or single mothers than for childless single individuals. This can be due to the fact that there is less variation in labor market behavior among childless singles or that non-participation corresponds more often to demand-side constraints (rather than to voluntary choice) in their case. This argument equally applies to single men-yet the fit of labor supply model for married men should be overall better when male and female decisions are jointly estimated. Second, estimates stemming from natural experiments are also limited for this group, given the fact that most welfare reforms in Anglo-Saxon countries concerned individuals or households with children (see the discussion in Bargain and Doorley 2011). The few available estimates point to very small elasticities. ${ }^{13}$ For both men (married or single) and childless single women, estimates are not only small but also very similar between studies for each country. This small variance across studies is illustrated in Fig. 2 (top-right quadrant for men and bottom-left quadrant for childless single women). Nonetheless, these mean values may hide much variation in participation responses across different wage or income groups, with important implications for welfare analysis as suggested by Eissa and Liebman (1996) and confirmed for single individuals in Bargain et al. (2014).

\subsection{Income elasticities}

Most studies show negative income elasticities of labor supply, i.e., leisure (or non-market time) is a normal good. Yet, positive income elasticities are encountered in some studies, which include Kuismanen (1997) for Finland, Flood and MaCurdy (1992) for Sweden, van Soest (1995) for the Netherlands, and Blau and Kahn (2007) and Cogan (1981) for the USA. Also, despite being generally small, income elasticities vary across countries. Blundell and MaCurdy (1999) report that variation between studies regarding income elasticity appears to be greater than the corresponding variation with respect to wage 
elasticities. This is not confirmed in the series of estimates produced for 18 countries in Bargain et al. (2014) and neither in Tables 1, 2, and 3 here. Note that very few estimates of income effects are available for single individuals.Since income effects tend to be small, in most cases, compensated and uncompensated wage elasticities are almost identical and very few studies report both. While Bargain et al. (2014) report both types of elasticities, they do not find big differences, in line with their small income elasticities.

\section{Meta-analysis of the relative contributions of time changes and estimation methods}

In Tables 1, 2, and 3 and Figs. 1 and 2, we have observed much variation across studies in the size of wage elasticities, especially for married women and single mothers. Studies that rule out differences in estimation methods and data years show that little of this is due to genuine variation in preferences across countries (Bargain et al. 2014). We investigate here the relative roles of time variation and estimation methods in explaining the diversity of elasticity estimates.

\subsection{Graphical analysis}

We begin our investigation of the respective contributions of time change versus estimation methods by relying on simple visual inspections. We focus our meta-analysis on married women and single mothers.

Time trends In Fig. 3 (left quadrant), we plot estimates by year of data collection as specified in surveyed studies (Tables 1, 2, and 3). A very clear declining trend emerges, showing in particular a concentration of low elasticities since the end of the 1990s, high elasticities in the 1970s, and more variation in between. This pattern can be observed for

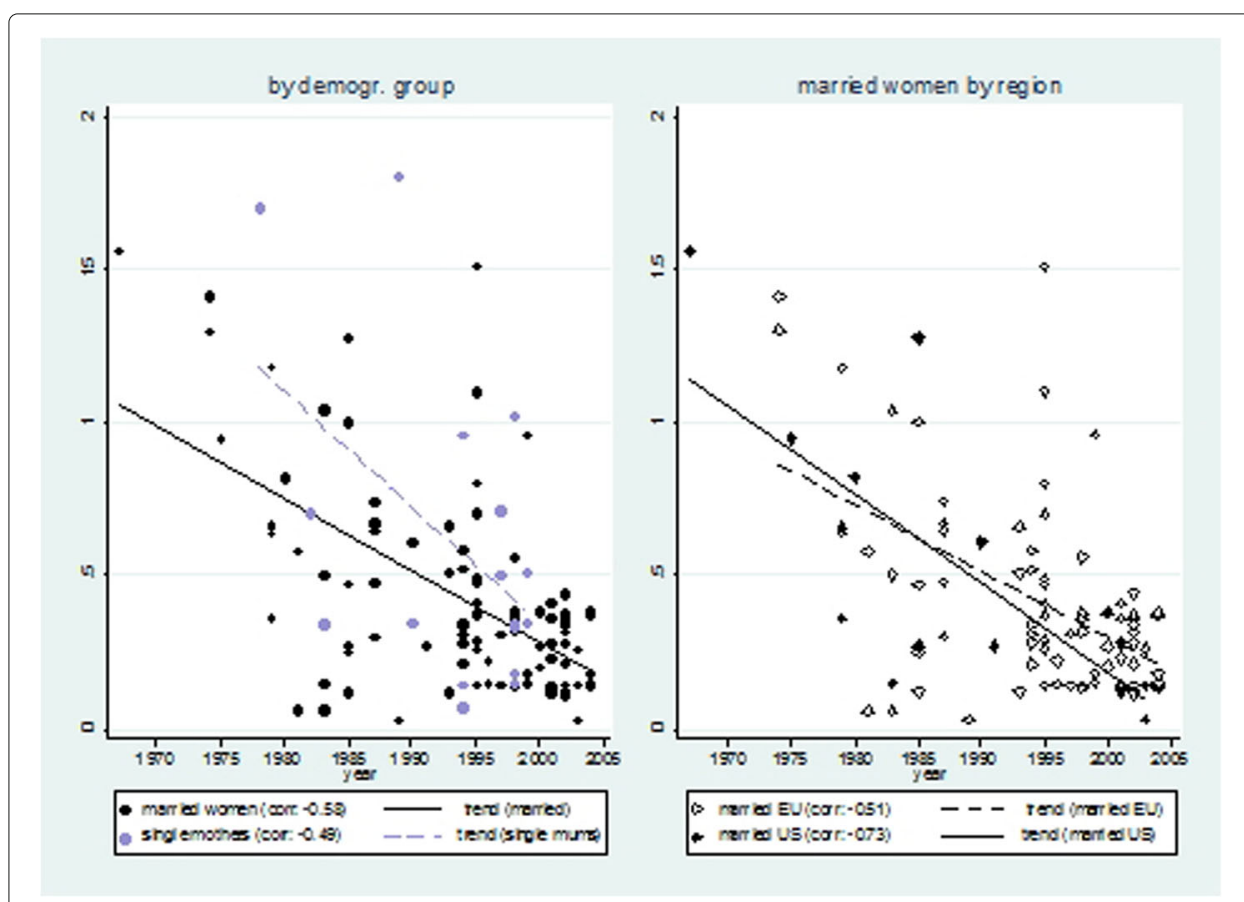

Fig. 3 Time trend in wage elasticities of married women and single mothers 
both married women and single mothers. Given the small number of US studies reporting estimates for the latter group, we focus on married women in the right quadrant of Fig. 3 where we distinguish between EU and US estimates. The trend is similar in both regions, with a strong negative correlation between the period of observation and the elasticity level. ${ }^{14}$ These findings tend to corroborate the result of Heim (2007) and Blau and Kahn (2007), who show that the labor supply elasticity of married women has strongly declined over time in the USA. They suggest a change in work preferences of women as possible explanation, but other explanations are possible (change in childcare policies, in domestic technology, etc.). Our results reveal that a similar trend exist for EU countries. Yet, estimates in Heim (2007) and Blau and Kahn (2007) rely on a uniform approach for the different periods while our meta-analysis possibly mixes time effects and changes in modeling and estimation methods over time.

Estimation methods To investigate this point further, let us go back to survey Tables 1, 2, and 3. A first observation is that early evidence using the Hausman technique points to relatively large own-wage elasticities for married women, sometimes close to 1, or even larger, for instance, in early studies for France, Germany, Italy, or the UK. In contrast, recent evidence based on discrete-choice models shows more modest elasticities for this demographic group, in a range between .1 and .5, with some exceptions. In Table 3, we observe a similar pattern for the USA, with very large estimates in early studies, including Hausman (1981), and more modest and comparable elasticities in the recent studies (total hour-wage elasticities ranging between .2 and .4, for instance, in Eissa and Hoynes 2004 or Heim 2007, 2009). Hence, we can conjecture that the estimation method explains time differences.

What are the possible underlying mechanisms? With the Hausman approach, the combination of restrictive functional forms (linear labor supply) and estimation methods that impose theoretical consistency of the labor supply model everywhere in the sample (global satisfaction of Slutsky conditions) can lead to biased estimates and possibly an overstatement of work incentives, as discussed above. In addition, this approach is more sensitive to the model specification which may explain the large variance in estimates from the 1970s and 1980s. Mroz (1987) shows how the wage effects of married women's labor supply varies dramatically depending on whether and how one controls for non-random selection into work as well as to alternative exclusion restrictions in the instrument set for wages. Bourguignon and Magnac (1990) discuss the sensitivity of their results to the model specification and show that the Hausman approach can lead to implausibly high elasticity values, as they find in some of their specifications. Drawn from our tables, we can see for instance that married women's wage elasticity obtained with the Hausman approach vary from .28 (Triest 1990) to .97 (Hausman 1981) in the USA, even when similar periods are considered (1983 and 1975 in these two studies, respectively). For France, estimates for married women are also very high with the basic Hausman model but almost zero when introducing fixed costs (cf. Bourguignon and Magnac 1990). Estimates obtained with discrete-choice models are somewhat more comparable from one study to the next. Yet, there are still differences, which are more likely driven by selection criteria (for France, high elasticities are found for families with children in Choné et al. 2003) and alternative specifications of discrete-choice models (for instance, the degree of flexibility of the model, see Bargain 2009). 
Time trends versus estimation methods To further investigate whether elasticities truly decline over time or whether this pattern is due to changes in estimation methods, we compare the trends in elasticities obtained with the two main methods. ${ }^{15}$ That is, Fig. 4 plots against data years the estimates obtained with continuous models (which rely mainly on the Hausman approach) and those from discrete-choice models (as recently used in many policy papers). Graphs in the upper panels show that the former was mainly used before 1990 while the latter approach took over in the 1990s and 2000s.

For continuous models, there are nonetheless some observations in the more recent years so that we can suggest tentative interpretations. For our group of interest, and whether single mothers are included (upper panel, right) or not (left), the time shrinking elasticity hypothesis is verified over all estimates relying on the Hausman approach. The linear correlation between time and elasticity size is around -.55 for married women with or without single mothers. When differentiating between regions and focusing on married women, in the lower panels of Fig. 4, this meta-analysis corroborates the findings in Heim (2007) and Blau and Kahn (2007) for the USA (both studies relying on a Hausmantype approach) and also finds a similar pattern for EU countries. Yet, it is noticeable that there are very few estimates based on the Hausman model for the period after 1990 in the EU, so the result is more fragile than for the USA.

If we turn to estimates from discrete-choice models, the upper graphs show fewer points of observations available before the 1990s. There is nonetheless a negative linear

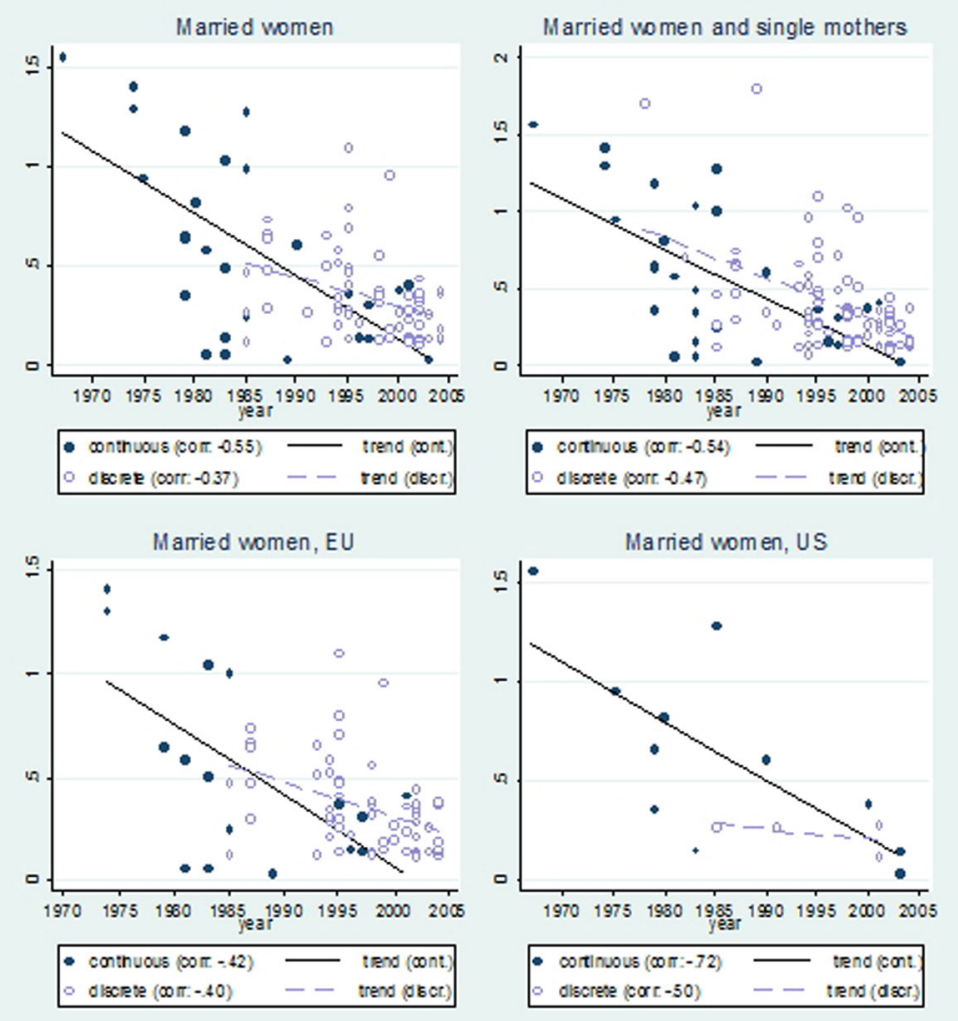

Fig. 4 Time trend in wage elasticities by broad estimation methods 
correlation between years and estimates due to the high density of very low estimates in the years 2000s. Over all years, this correlation is -.37 for married women (going down to -.22 if German estimates, which are numerous in this period, are taken out) and -.47 when adding single mothers ( -.40 without Germany). If we take out the $2000 \mathrm{~s}$, it goes down to -.08 for married women and -.34 when adding single mothers (Germany does not change the picture this time). For a comparison, the correlation between years and Hausman estimates remain at -.47 over this subperiod (with or without single mothers). The lower panel confirms results for the EU while too few estimates based on discrete-choice models exist for the USA to attempt any interpretation.

\subsection{Meta-regression analysis}

To disentangle time changes and estimation methods, we finally proceed with simple meta-estimations. ${ }^{16}$ Focusing first on married women, we regress elasticity values for a set of simple model characteristics. ${ }^{17}$ Results are reported in Table 4. Reflecting our discussion above on the limits of our observations, the first columns focus on data years for which we can find some common support in the use of the two empirical methods. That is, we restrict our sample to a period starting with the data year of the first estimate obtained with a discrete-choice model (estimates on CPS 1985 in Eissa and Hoynes 2004 and on the Dutch Labor Mobility Survey 1985 in van Soest et al. 1990). The main conclusion is that estimation periods ("year") turn out to play a significant role. An additional year decreases wage elasticities of married women by around .013, which amounts to a decrease of .31 over a period of 24 years (the duration considered in Heim 2007). This conclusion holds whether we include the estimation method (a "discrete model" dummy) or not. In contrast, the estimation method is itself broadly insignificant. That is, the "overestimation" due to the Hausman model is not particularly visible when time effects are taken into account.

Results are basically unchanged whether we consider total hour elasticities or participation response alone (4th and 5th columns). The same is true if we focus on EU estimates only (6th column) or if we extend the period to all the years in our sample of estimates for married women (last three columns). ${ }^{18}$ In this last set of results, the estimation method alone has a significant effect which likely captures time trends due to the correlation between period and method. Indeed, this effect disappears when we account for years while years themselves are unaffected by the inclusion of the estimation method dummy.

Hence, despite the limited common support, these meta-regressions tend to confirm the shrinking elasticity hypothesis while estimation methods do seem to affect elasticity size much. Up to this point, we have considered only linear time effects. Using all data years, additional (unreported) regressions with quadratic trend in years show that a larger decline in elasticities occurred in the early period. This is confirmed by a regression with dummies for periods 1967-1984 and 1985-1995, which shows that elasticities were respectively .497 ( $p$ value of 0.001$)$ and .145 ( $p$ value of 0.053 ) larger than those in the reference period of 1996-2004. When using only post-1985 data (common support), a similar approach confirms that elasticities of the period 1985-1995 were .143 larger ( $p$ value of 0.013 ) than those in the most recent period. This conveys that much of the changes in female labor force participation had already taken place in the early period.

Turning back to Table 4, we see that addition controls corresponding to modeling options do not play a significant role. An exception is the use of desired rather than 
Table 4 Meta-regression of married women's wage elasticities

\begin{tabular}{|c|c|c|c|c|c|c|c|c|c|}
\hline \multirow[b]{2}{*}{ Model } & \multicolumn{6}{|c|}{ On years with common support for the use of both Hausman and discrete models ${ }^{\mathrm{a}}$} & \multirow{2}{*}{\multicolumn{3}{|c|}{$\begin{array}{l}\text { On all years }{ }^{b} \\
\text { All elasticities }\end{array}$}} \\
\hline & & All elasticities & & Participation elasticities & Hourelasticities & Without the US & & & \\
\hline \multirow[t]{2}{*}{ Year } & & $-0.013^{* * *}$ & $-0.013^{* * *}$ & $-0.012^{* *}$ & $-0.012^{*}$ & $-0.013^{* *}$ & & $-0.024^{* * *}$ & $-0.024^{* * *}$ \\
\hline & & $(.005)$ & $(.005)$ & $(.007)$ & $(.006)$ & $(.005)$ & & $(.004)$ & $(.004)$ \\
\hline \multirow[t]{2}{*}{ Discrete model } & 0.012 & & 0.013 & 0.170 & -0.007 & 0.043 & $-0.236^{* *}$ & & -0.012 \\
\hline & $(.083)$ & & $(.079)$ & $(.251)$ & (.098) & $(.090)$ & $(.095)$ & & $(.089)$ \\
\hline \multirow[t]{2}{*}{ Desired hours } & $0.258^{* * *}$ & $0.185^{* *}$ & $0.185^{* *}$ & 0.086 & $0.837^{* *}$ & $0.177^{* *}$ & $0.253^{* *}$ & 0.120 & 0.121 \\
\hline & $(.077)$ & $(.078)$ & $(.079)$ & $(.114)$ & $(.106)$ & (.083) & (.109) & $(.094)$ & $(.095)$ \\
\hline \multirow[t]{2}{*}{ Joint decision } & -0.068 & -0.022 & -0.026 & -0.087 & 0.024 & -0.013 & -0.043 & 0.005 & 0.008 \\
\hline & $(.063)$ & $(.057)$ & $(.062)$ & $(.085)$ & (.088) & $(.067)$ & $(.083)$ & $(.066)$ & $(.071)$ \\
\hline \multirow[t]{2}{*}{ Fixed $\cos ^{\mathrm{c}}$} & 0.007 & 0.027 & 0.025 & -0.024 & 0.069 & 0.014 & 0.007 & 0.039 & 0.041 \\
\hline & $(.059)$ & $(.055)$ & $(.057)$ & $(.074)$ & $(.082)$ & $(.060)$ & $(.082)$ & $(.067)$ & $(.070)$ \\
\hline \multirow[t]{2}{*}{ US } & -0.050 & -0.049 & -0.045 & -0.083 & 0.006 & & -0.015 & -0.044 & -0.046 \\
\hline & $(.088)$ & $(.081)$ & $(.084)$ & $(.150)$ & (.108) & & (.098) & $(.080)$ & $(.083)$ \\
\hline \multirow[t]{2}{*}{ Constant } & $0.341^{* * *}$ & $0.470^{* * *}$ & $0.462^{* * *}$ & 0.323 & $0.454^{* * *}$ & $0.439^{* * *}$ & $0.569^{* * *}$ & $1.025^{* * *}$ & $1.025^{* * *}$ \\
\hline & $(.068)$ & $(.062)$ & $(.079)$ & $(.283)$ & $(.089)$ & $(.085)$ & $(.069)$ & $(.096)$ & $(.096)$ \\
\hline $\mathrm{Nb}$ of observations & 75 & 75 & 75 & 32 & 43 & 67 & 90 & 90 & 90 \\
\hline R2 & 0.16 & 0.24 & 0.24 & 0.27 & 0.30 & 0.22 & 0.15 & 0.40 & 0.40 \\
\hline
\end{tabular}

Note: we regress elasticity values on modeling choices using estimates on a data from 1985 to 2004 and ${ }^{b}$ data from 1967 to 2004

"Work cost specification in discrete models

$*^{* *}$ and ${ }^{* * *}$ indicate significance levels at $1 \%, 5 \%$ and $10 \%$ respectively 
observed hours, which inflates hour-wage elasticities. This necessarily reflects the role of demand-side or institutional constraints on working time and the fact that models estimated on observed work duration do underestimate potential labor supply responses. Finally, Table 5 reports meta-estimates for a larger group including married women and single mothers. Results are qualitatively very similar to those for married women alone. The only visible difference is the slightly large time effect obtained for participation elasticities compared to hour elasticities. This echoes our discussion in the survey (Section 3.2) about the importance of the extensive margin for the group of single mothers, especially in the UK.

\section{Conclusions}

In this paper, we provide an extensive survey of studies estimating static labor supply elasticities for Western Europe and the USA. We do not only confirm most of the usual stylized facts from older reviews but also derive original results concerning the variation in labor supply responses across studies. While Bargain et al. (2014) show that international heterogeneity in work preference matters but is small, we investigate the role of two factors that greatly influence the variance in elasticity size across studies, namely the time period and the estimation method. It is often suspected that large elasticities found in the literature are due to either labor market conditions of the 1970s and 1980s (and notably more intermittent female labor market participation than in the recent period) and/or to the use of a Hausman-type of model estimation (which tend to overestimate responses in some of its specifications). More recent estimates based on structural discrete-choice models with tax-benefit simulations show smaller estimates and relatively more similarity across studies. Our meta-analysis confirms that elasticities for married women and single mothers have indeed declined over time in the USA (as shown in Heim 2007 and Blau and Kahn 2007) and also in the EU. This time effect reflects a possible change in work preferences (and a stronger attachment of women to the labor market), in social preferences (embodied in public childcare policies), and in domestic technologies. In contrast, we find less compelling evidence that the choice of estimation method explains much variation in estimates.

Further validation of these results can be obtained at the price of an extensive estimation of discrete-choice models over the long period. This would also facilitate tests of the explanations underlying the shrinking elasticity hypothesis of Heim (2007) and Blau and Kahn (2007). In particular, a change in preferences over the past four decades can be tested directly on the preference parameters revealed by discrete-choice model estimations. This would require data since at least the early 1980s (and if possible since the 1970s) and tax-benefit simulations for all the years since these periods, in order to use policy change for robust identification. This research avenue is tedious but clearly feasible for some countries for which comparable data are available over the long run (the USA, the UK, and Germany in particular).

Our results have important implications as labor supply elasticities are key parameters when evaluating or designing optimal tax-benefit policies. For instance, Diamond and Saez (2011) use an elasticity of 0.25 to derive an optimal top marginal tax rate of $72.7 \%$. However, an elasticity of 0.6 reduces the optimal tax rate to $52.6 \%$, bringing it closer to actually observed values. However, our study shows that using a single number obtained from one study for such calculations might be misleading for several reasons. First of all, 
Table 5 Meta-regression of married women and single mothers' wage elasticities

\begin{tabular}{|c|c|c|c|c|c|c|c|c|c|}
\hline \multirow[b]{2}{*}{ Model } & & \multicolumn{5}{|c|}{ On years with common support for the use of both Hausman and discrete models ${ }^{a}$} & \multirow{2}{*}{\multicolumn{3}{|c|}{$\begin{array}{l}\text { On all years }{ }^{\mathrm{b}} \\
\text { All elasticities }\end{array}$}} \\
\hline & & All elasticities & & Participation elasticities & Hourelasticities & Without the US & & & \\
\hline Year & & $\begin{array}{l}-0.020^{* * *} \\
(.006)\end{array}$ & $\begin{array}{l}-0.020^{* * *} \\
(.006)\end{array}$ & $\begin{array}{l}-0.033^{* * *} \\
(.010)\end{array}$ & $\begin{array}{l}-0.011^{*} \\
(.006)\end{array}$ & $\begin{array}{l}-0.018^{* * *} \\
(.006)\end{array}$ & & $\begin{array}{l}-0.025^{* * *} \\
(.004)\end{array}$ & $\begin{array}{l}-0.028^{* * *} \\
(.004)\end{array}$ \\
\hline Discrete model & $\begin{array}{l}0.004 \\
(.097)\end{array}$ & & $\begin{array}{l}0.042 \\
(.092)\end{array}$ & $\begin{array}{l}-0.230 \\
(.279)\end{array}$ & $\begin{array}{l}0.019 \\
(.083)\end{array}$ & $\begin{array}{l}0.121 \\
(.108)\end{array}$ & $\begin{array}{l}-0.148^{*} \\
(.090)\end{array}$ & & $\begin{array}{l}0.122 \\
(.088)\end{array}$ \\
\hline Desired hours & $\begin{array}{l}0.225^{* *} \\
(.103)\end{array}$ & $\begin{array}{l}0.131 \\
(.100)\end{array}$ & $\begin{array}{l}0.127 \\
(.101)\end{array}$ & $\begin{array}{l}-0.093 \\
(.184)\end{array}$ & $\begin{array}{l}0.246^{* *} \\
(.101)\end{array}$ & $\begin{array}{l}0.133 \\
(.099)\end{array}$ & $\begin{array}{l}0.215^{*} \\
(.126)\end{array}$ & $\begin{array}{l}0.104 \\
(.109)\end{array}$ & $\begin{array}{l}0.083 \\
(.109)\end{array}$ \\
\hline Fixed $\operatorname{cost}^{\mathrm{c}}$ & $\begin{array}{l}-0.065 \\
(.071)\end{array}$ & $\begin{array}{l}-0.015 \\
(.066)\end{array}$ & $\begin{array}{l}-0.022 \\
(.068)\end{array}$ & $\begin{array}{l}-0.113 \\
(.111)\end{array}$ & $\begin{array}{l}0.053 \\
(.074)\end{array}$ & $\begin{array}{l}-0.041 \\
(.067)\end{array}$ & $\begin{array}{l}-0.105 \\
(.086)\end{array}$ & $\begin{array}{l}-0.003 \\
(.073)\end{array}$ & $\begin{array}{l}-0.024 \\
(.074)\end{array}$ \\
\hline US & $\begin{array}{l}0.052 \\
(.103)\end{array}$ & $\begin{array}{l}0.024 \\
(.094)\end{array}$ & $\begin{array}{l}0.035 \\
(.097)\end{array}$ & $\begin{array}{l}-0.099 \\
(.181)\end{array}$ & $\begin{array}{l}-0.001 \\
(.106)\end{array}$ & & $\begin{array}{l}0.027 \\
(.104)\end{array}$ & $\begin{array}{l}-0.028 \\
(.087)\end{array}$ & $\begin{array}{l}-0.001 \\
(.089)\end{array}$ \\
\hline Constant & $\begin{array}{l}0.372^{* * *} \\
(.091)\end{array}$ & $\begin{array}{l}0.596^{* * *} \\
(.076)\end{array}$ & $\begin{array}{l}0.565^{* * *} \\
(.102)\end{array}$ & $\begin{array}{l}1.053^{* * *} \\
(.315)\end{array}$ & $\begin{array}{l}0.444^{* * *} \\
(.088)\end{array}$ & $\begin{array}{l}0.478^{* * *} \\
(.108)\end{array}$ & $\begin{array}{l}0.566^{* * *} \\
(.078)\end{array}$ & $\begin{array}{l}1.111^{* * *} \\
(.108)\end{array}$ & $\begin{array}{l}1.106^{* * *} \\
(.107)\end{array}$ \\
\hline $\mathrm{Nb}$ of observations & 90 & 90 & 90 & 42 & 48 & 79 & 108 & 108 & 108 \\
\hline R2 & 0.06 & 0.17 & 0.18 & 0.29 & 0.26 & 016 & 0.08 & 0.33 & 0.34 \\
\hline
\end{tabular}

Note: we regress elasticity values on modeling choices using estimates on a data from 1985 to 2004 and ${ }^{b}$ data from 1967 to 2004

cWork cost specification in discrete models

${ }^{c}$. Work cost specification in discrete models ${ }^{* * *}$ indicate significance levels at $1 \%, 5 \%$ and $10 \%$ respectively 
it should be emphasized that there is not one "right" elasticity. A labor supply elasticity per se is not a deep structural parameter and depends on many factors including the country (wage distribution, institutions, labor market characteristics), the demographic group under investigating, and the time period, among others. It seems that countryspecific preferences are not the primary concern. From the amount of evidence collected and discussed in this paper, policy simulations should rather make use of estimates that are derived from data collected close to the time period that analysts are looking at and for the particular demographic groups affected by the policies under study. Making the "right" choice is not easy, and we suggest using a range of "plausible" values for sensitivity checks. One may want to avoid using reduced-form estimates which lack external validity, if simulations imply important changes in a wide range of policies. As for estimations of new elasticities (our survey has shown that there are very few estimates available for some countries and some periods), one may prefer discrete-choice models than other structural approaches like the Hausman model. We found not clear evidence for significant average differences in elasticity sizes, and the critiques of the Hausman model might be overstated. However, as discussed in Section 2, discrete models usually provide a more comprehensive setting that accounts for the complete set of fiscal and social incentives, for explicit intensive versus extensive margins of response, and for joint decisions within couples. Estimates in our survey also show less variability across studies, conveying that the Hausman approach is too sensitive to specification choices. Whatever the structural approach used for policy simulations, however, better identification strategies may be required than tax non-linearities in cross-sectional data. Identification of behavioral parameters may rely on exogenous variation from tax reforms (as in Blundell et al. 1998), experimental data, or quasi-experiments like discontinuities (Bargain and Doorley 2016).

\section{Endnotes}

${ }^{1}$ Bargain et al. (2014) conduct an estimate labor supply elasticities for 17 European countries and the USA, separately by gender and marital status. Measurement differences are netted out by using a harmonized empirical approach and comparable data sources and years. Bargain et al. (2014) find that own-wage elasticities are relatively small and much more uniform across countries than previously thought. Differences exist nonetheless and are found not to arise from different tax-benefit systems or demographic compositions across countries.

${ }^{2}$ See Chetty (2012) for an interesting discussion about the potential bias in the estimation of labor supply in the presence of optimization frictions. To cope with this problem, Chetty provides bounds on the structural elasticities derived from reduced-form estimates that might suffer from optimization friction bias. The derived bounds imply that frictions affect intensive margin elasticities much more than extensive margin elasticities.

${ }^{3}$ Arguably, these other margins partly relate to responses not directly pertaining to productive behavior, like tax evasion and optimization. In this regard, hours of work still constitute an interesting benchmark. Another margin is work effort that may affect wage rates. In the short run, however, hours and participation are the only variables of adjustment for a majority of workers.

${ }^{4}$ These elasticities are much larger than in microeconomic studies (e.g., Prescott 2004). Several reasons have been suggested for this: the use of representative agents and difficulties around an aggregation theory when heterogeneity matters (see Blanchard 2006), 
the existence of a social multiplier whereby the utility from not working is increasing in the number of people who do not work (see Alesina et al. 2005), and factors related to the timing and the nature of labor supply adjustments in the presence of frictions (Chetty et al. 2011).

${ }^{5}$ Another approach is the reconvexification of the budget set. For instance, to estimate the labor supply of married women on 1985 French data, Bourguignon and Magnac (1990) use the Hausman technique and eliminate minor non-convexities by replacing the budget set by its convex envelope. This approach is not possible for later years as the implementation of a minimum income scheme in 1988 has introduced high nonconvexity in the budget constraint. Similar non-convexities arise in all countries with substantial means-tested transfers.

${ }^{6}$ See Eklöf and Sacklén (2000) for a critical discussion of the MaCurdy critique.

${ }^{7}$ This issue is shared with the literature on the elasticity of taxable income, whereby results are sensitive to the type of reforms exploited for identification (Saez et al. 2012). Indeed, control group definition follows from their income level, so that specific preferences are identified and results cannot be extrapolated. For instance, changes in tax rates (tax credits) identify the preferences of high (low)-income groups and may not be generalized to the whole population.

${ }^{8}$ Things are changing in the recent period. For France, for instance, some studies have recently used tax-benefit changes to evaluate the responsiveness of the labor force, including the introduction of a small tax credit (Stancanelli 2008), time change in income tax schedule (Carbonnier 2008), changes in the possibility to cumulate welfare payment for lone mothers and earnings (González 2008), and age condition on children for a replacement income targeted at low-income mothers who opt for full-time childcare (Piketty 1998). RD estimations using age conditions on the level of social assistance program are also used in Bargain and Doorley (2011), in a similar way as Lemieux and Milligan (2008) for Canada.

${ }^{9}$ Note that few papers report uncompensated elasticities. One reason for this is that income effects are often very small and, hence, compensated and uncompensated elasticities are almost identical. This is confirmed by the findings in Bargain et al. (2014) - one of the few studies reporting both types of elasticities.

${ }^{10}$ Note that almost all papers report elasticities with respect to the gross wage. Bargain et al. (2014) show that net wage elasticities are-mechanically-slightly larger than gross wage elasticities.

${ }^{11}$ Note that a variety of different elasticities is used in different studies. For example, there are compensated and uncompensated wage elasticities, different ways in which the tax system is (or is not) accounted for, elasticities with respect to gross wage rates or net wage rates, etc. Moreover, it also may matter how elasticities are aggregated over the group of interest. Some studies present the elasticity for a benchmark (average) observation, others an elasticity of total hours of work in the whole group for a uniform wage rate increase, some even present an average of the elasticities for all observations. However, most studies report uncompensated elasticities with respect to the gross wage for total hours of work. We have tried to harmonize the definitions as much as possible when choosing the elasticities.

${ }^{12}$ Note that not all papers report elasticities in the same way. It was our task to make sure that we identified the same parameter-namely the uncompensated wage elasticity 
or the income elasticity-from each paper. We have always chosen the author's preferred estimate in case they reported several estimates (for example, as robustness checks).

${ }^{13}$ For instance, Euwals and van Soest (1999) report wage elasticities for childless single individuals in the Netherlands of around .10-.11. For Germany, a series of studies report estimates between .10 and .36 for childless single men and women.

${ }^{14}$ We also find similar patterns when looking separately at hour-wage elasticities (correlation of -.59 with observation years) and participation-wage elasticities (correlation of -.54 ) for married women.

${ }^{15}$ Further potentially important factors are the treatment of wages in the estimation procedure (Löffler et al. 2014) and the choice of the reference tax system for the benchmark.

${ }^{16}$ Note that-like all meta-regressions-our analysis is not identifying causal effects since one can think of several potentially omitted variables.

${ }^{17}$ Note that we cannot include all possible aspects of model specification in our paper since there are too many dimensions and to few observations. For example, Löffler et al. (2014) investigate the role of the treatment of wages for the size of elasticities. In order to do this, the authors estimate 3500 elasticities using a discrete-choice framework with all possible permutations of model choices. Unfortunately, such a variation is missing in the estimates collected for our study.

${ }^{18}$ We do not report similar estimations for the USA only given the small number of observations in this case.

\section{Acknowledgements}

We would like to thank Pierre Cahuc and two anonymous referees, as well as Herwig Immervoll, Max Löffler, Kristian Orsini, Sebastian Siegloch, and Carina Woodage for the useful comments on earlier drafts. The usual disclaimer applies. Responsible editor: Pierre Cahuc.

\section{Funding}

None.

\section{Competing interests}

The IZA Journal of Labor Economics is committed to the IZA Guiding Principles of Research Integrity. The authors declare that they have observed these principles.

\section{Author details}

${ }^{1}$ Aix-Marseille University (Aix-Marseille School of Economics), CNRS \& EHESS, Marseille, France. ${ }^{2}$ IZA, Bonn, Germany.

${ }^{3}$ ZEW, University of Mannheim, IZA and CESifo, L7, 1, 68161 Mannheim, Germany.

Received: 24 May 2016 Accepted: 7 September 2016

Published online: 31 October 2016

\section{References}

Aaberge R, Dagsvik J, Strom S (1995) Labor supply responses and welfare effects of tax reforms. Scand J Econ 97(4):635-659

Aaberge R, Colombino U, Strøm S (1999) Labour supply in Italy: an empirical analysis of joint household decisions, with taxes and quantity constraints. J Appl Econ 14:403-422

Aaberge R, Colombino U, Wennemo T (2002) Heterogeneity in the elasticity of labour supply in Italy and some policy implications, WP CHILD. \#21/2002

Aaberge R, Colombino U, Strøm S (2004) Do more equal slices shrink the cake? An empirical investigation of tax-transfer reform proposals in Italy. J Popul Econ 17:767-785

Aaberge R, Colombino U (2012) Accounting for family background when designing optimal income taxes: a microeconometric simulation analysis. J Popul Econ 25(2):41-761

Alesina A, Glaeser E, Sacerdote B (2005) Work and leisure in the US and Europe: why so different. NBER Macroeconomic Annual

Andrén T (2003) A structural model of childcare, welfare, and the labor supply of single mothers. Labour Econ 10(2):133-147

Arellano M, Meghir C (1992) Female labour supply and on the job search: an empirical model estimated using complementary data sets. Rev Econ Stud 59:537-559 
Arrufat JL, Zabalza A (1986) Female labour supply with taxation, random preferences and optimization errors. Econometrica 54(1):47-63

Bargain O (2009) Flexible labour supply models. Econ Lett 105:103-105

Bargain O, Orsini K (2006) In-work policies in Europe: killing two birds with one stone Labour Econ 13:667-697

Bargain O, Caliendo M, Haan P, Orsini K (2010) Making work pay in a rationed labour market. J Popul Econ 23(1):323-351

Bargain O, Doorley K (2011) Caught in the trap? Welfare's disincentive and the labor supply of single men. J Public Econ 95(9-10):1096-1110

Bargain, O, Doorley K (2016) Putting structure on the RD design: social transfers and youth inactivity in France. IZA Discussion Papers: 7508. Forthcoming in the Journal of Human Resources (published online before print: doi:10.3368/jhr.52.4.1115-7510R, J Human Resources July 7, 2016 1115-7510R)

Bargain O, Orsini K, Peichl A (2014) Comparing labor supply elasticities in Europe and the US: new results. J Human Resources 49(3):723-838

Bingley P, Walker I (1997) The labour supply, unemployment and participation of lone mothers in in-work transfer programmes. Econ J 107:1375-1390

Bishop K, Heim B, Mihaly K (2009) Single women's labor supply elasticities: trends and policy implications. Ind Labor Relations Rev 63(1):146-168

Blanchard O (2006) Discussion of "Do taxes explain European employment? Indivisible labor, human capital, lotteries and savings" (by L. L.ungqvist and T. Sargent). NBER Macroeconomics Conference:2006

Blau F, Kahn L (2007) Changes in the labor supply behavior of married women: 1980-2000. J Labor Econ 25:393-438

Bloemen HG (2009) An empirical model of collective household labour supply with non-participation. Econ J 120:183-214

Bloemen HG (2010) Income taxation in an empirical collective household labour supply model with discrete hours, IZA Discussion Papers. No:4697

Blomquist S (1983) The effect of income taxation on the labor supply of married men in Sweden. J Public Econ 22:169-197

Blomquist S, Hansson-Brusewitz U (1990) The effect of taxes on male and female labor supply in Sweden. J Hum Resour 25:317-357

Blomquist S, Newey W (2002) Nonparametric estimation with nonlinear budget sets. Econometrica 70(6):2455-2480

Blundell RW, Walker I (1986) A life cycle consistent empirical model of labour supply using cross section data. Rev Econ Stud 53:539-558

Blundell RW, Duncan A, Meghir C (1992) Taxation and empirical labour supply models: lone parents in the UK. Econ J 102:265-278

Blundell RW, Duncan A, Meghir C (1998) Estimating labor supply responses using tax reforms. Econometrica 66:827-861

Blundell RW, Ham J, Meghir C (1987) Unemployment and female labour supply. Econ J 97:44-64

Blundell RW, MaCurdy T (1999) Labor supply: a review of alternative approaches. In: Ashenfelter and Card (eds) Handbook of labor economics, vol.3A, ch. 27. Elsevier, North-Holland

Blundell RW, Duncan A, McCrae J, Meghir C (2000) The labour market impact of the working families' tax credit. Fiscal Studies 21(1):75-103

Blundell RW, MaCurdy T, Meghir C (2007) Labor supply models: unobserved heterogeneity, nonparticipation and dynamics, Handbook of Econometrics, Volume 6A Chapter 69 Heckman and Leamer eds

Bonin H, Kempe W, Schneider H (2002) Household labor supply effects of low-wage subsidies in Germany. J Appl Social Sci Stud 123:199-208

Bourguignon F, Magnac T (1990) Labour supply and taxation in France. J Hum Resour 25:358-389

Brewer M, Duncan A, Shephard A, Suarez MJ (2006) Did the working families' tax credit work? The impact of in-work support on labour supply in Great Britain. Labour Econ 13(6):699-720

Brink A, Nordblom K, Wahlberg R (2007) Maximum fee versus child benefit: a welfare analysis of Swedish child-care fee reform. Int Tax Public Finance 14(4):457-480

Callan T, van Soest A (1996) Family labour supply and taxes in Ireland. ESRI working paper

Callan T, van Soest A, Walsh J (2009) Tax structure and female labour supply: evidence from Ireland. Labour 23(1):1-35

Carbonnier C (2008) Spouse labor supply: fiscal incentive and income effect, evidence from French fully joint income tax system, working paper THEMA

Chetty R (2012) Bounds on elasticities with optimization frictions: a synthesis of micro and macro evidence on labor supply. Econometrica 80(3):969-1018

Chetty R, Guren A, Manoli D, Weber A (2011) Are micro and macro labor supply elasticities consistent? A review of evidence on the intensive and extensive margins. Am Econ Rev 101:471-75

Choné P, Le Blanc D, Robert-Bobée I (2003) Female labor supply and child care in France. CREST working paper

Clauss M, Schnabel R (2006) Distributional and behavioural effects of the German labour market reform. ZEW working paper

Cogan J (1981) Fixed cost and labour supply. Econometrica 49:945-964

Colombino U, del Boca D (1990) The effect of taxes on labor supply in Italy. J Hum Resour 25:390-414

Crespo L (2006) Estimation and testing of household labour supply models: evidence from Spain, mimeo

Dagsvik JK, Jia Z, Orsini K, van Camp G (2011) Subsidies on low-skilled workers' social security contributions: the case of Belgium. Empir Econ 40(3):779-806

Dagsvik JK (2006) Strøm, S. Sectoral labor supply, choice restrictions and functional form. J Appl Econ 21:803-826

Dearing H, Hofer H, Lietz C, Winter-Ebmer R, Wrohlich K (2007) Why are mothers working longer hours in Austria than in Germany? A comparative microsimulation analysis. Fisc Stud 28(4):463-495

Devereux P (2003) Changes in male labor supply and wages. Ind Labor Relat Rev 56:409-428

Devereux (2004) Changes in relative wages and family labor supply. J Human Resour 39:696-722

Diamond P, Saez E (2011) The case for a progressive tax: from basic research to policy recommendations. J Econ Perspect 25(4):165-190

Dickert S, Houser S, Scholz JK (1995) The earned income tax credit and transfer programs: a study of labor market and program participation. In: Poterba J (ed). Tax policy and the economy, 9th ed. MIT press, Cambridge. pp 1-50

Donni O, Moreau N (2007) Collective labor supply: a single-equation model and some evidence from French data. J Hum Resource 42(1):214-246 
Eissa N, Hoynes H (2004) Taxes and the labor market participation of married couples: the earned income tax credit. J Public Econ 88(9-10):1931-1958

Eissa N, Liebman J (1996) Labor supply response to the earned income tax credit. Q J Econ 111(2):605-637

Eklöf M, Sacklén H (2000) The Hausman-MaCurdy controversy: why do the results differ across studies? J Hum Resour 35(issue 1):204-220

Ermisch J, Wright R (1991) Welfare benefits and lone parents' employment in Great Britain. J Hum Resour 26(3):424-45

Euwals R, van Soest A (1999) Desired and actual labor supply of unmarried men and women in the Netherlands. Labor Econ 6:95-118

Evers M, de Mooij R, van Vuuren D (2008) The wage elasticity of labour supply: a synthesis of empirical estimates. De Economist 156:25-43

Fernández-Val I (2003) Household labor supply: evidence for Spain. Investigaciones Econó 27(2):239-275

Flood L, MaCurdy T (1992) Work disincentive effects of taxes: an empirical study of Swedish men. Carnegie-Rochester Conference Series on Public Policy

Flood L, Hansen J, Wahlberg R (2004) Household labour supply and welfare participation in Sweden. J Hum Ressour 39(4):1008-1032

Frederiksen A, Graversen E, Smith N (2008) Overtime work, dual job holding and taxation. Res Labor Econ 28:25-55

Fuchs VR, Krueger AB, Poterba JM (1998) Economists' views about parameters, values, and policies: survey results in labor and public economics. J Econ Lit 36(3):1387-1425

Fuest C, Peichl A, Schaefer T (2008) Is a flat rate reform feasible in a grown-up democracy of Western Europe? A simulation study for Germany. Int Tax Public Financ 15(5):620-636

García I SuárezMJ (2003) Female labour supply and income taxation in Spain: the importance of behavioural assumptions and unobserved heterogeneity specification. Hacienda Pública Española / Revista de Economía Pública 164(1):9-27

González L (2008) Single mothers, welfare, and incentives to work. LABOUR 22(3):447-468

Haan P, Steiner V (2004) Distributional and fiscal effects of the German tax reform 2000-a behavioral microsimulation analysis. DIW discussion paper, 419, Berlin

Haan P, Uhlendorff A (2007) Intertemporal labor supply and involuntary unemployment. IZA Discussion Paper No:2888

Hall RE (1973) Wages, income and hours of work in the U.S. labor force. In: Cain and Watts (ed). Income maintenance and labor supply. Chicago, Markham

Hausman JA (1981) Labor supply. In: Aaron H, Pechman J (eds). How taxes affect economic behavior. The Brookings Institution, Washington DC. pp 27-72

Hausman, JA (1985a) The econometrics of nonlinear budget sets. Econometrica 53:1255-82

Hausman JA (1985b) Taxes and labor supply, Auerbach and Feldstein. In: Auerbach A, Feldstein M (eds). Handbook of public economics, vol. 1. North-Holland publishes, Amsterdam

Hausman JA, Ruud P (1984) Family labor supply with taxes. Am Econ Rev 74:242-248

Heckman JA (1993) What has been learned about labor supply in the past twenty years Am Econ Rev 83:1 16-121

Heim BT (2007) The incredible shrinking elasticities: married female labor supply, 1978-2002. J Hum Resour 42(4):881-918

Heim BT (2009) Structural estimation of family labor supply with taxes: estimating a continuous hours model using a direct utility specification. J Hum Resour 44(2):350-385

Heim, BT, Meyer BD (2003) Work costs and nonconvex preferences in the estimation of labor supply models. J Public Econ 88(11):2323-38

Hotz J, Scholz K (2003) The earned income tax credit. In: Moffitt R (ed). Means-tested transfer programs in the United States. The University of Chicago Press and NBER. pp 141-197

Hoynes $\mathrm{H}$ (1996) Welfare transfers in two-parent families: labor supply and welfare participation under AFDC-UP. Econometrica 64:295-332

Jenkins S (1992) Lone mothers' employment and full time work probabilities. Econ J 102(411):310-320

Kaiser H, Spahn P, van Essen U (1992) Income taxation and the supply of labour in West Germany. Jahrbü 209/1-2:87-105

Keane M, Moffitt R (1998) A structural model of multiple welfare program participation and labor supply. Int Econ Rev 39:553-589

Keane MP (2011) Labor supply and taxes: a survey. J Econ Lit 49(4):961-1075

Keane MP, Rogerson R (2012) Micro and macro labor supply elasticities: a reassessment of conventional wisdom. J Econ Lit 50(2):464-76

Killingsworth M, Heckman J (1986) Female labor supply: a survey. In: Ashenfelter O, Layard R (eds). Handbook of labor economics. North-Holland, Amsterdam Vol. 1

Kuismanen M (1997) Labour supply, unemployment and income taxation: an empirical application, Working Paper. In: Government Institute for Economic Research. University College London, Helsinki

Labeaga JM, Oliver X, Spadaro A (2008) Discrete choice models of labour supply, behavioural microsimulation and the Spanish tax reform. J Econ Inequal 6(3):247-273

Laroque G, Salanié B (2002) Labor market, institutions and employment in France. J Appl Econom 17:25-48

Lemieux T, Milligan K (2008) Incentive effects of social assistance: a regression discontinuity approach. J Econom 142(2):807-828

Löffler M, Peichl A, Siegloch S (2014) Structural labor supply models and wage exogeneity. IZA Discussion Paper No:8281

MaCurdy T (1992) Work disincentive effects of taxes: a reexamination of some evidence. Am Econ Rev Papers Proc 82:243-249

MaCurdy T, Green P, Paarsch H (1990) Assessing empirical approaches for analyzing taxes and labor supply. J Hum Resour 25:415-490

Mastrogiacomo M, Bosch NM, Gielen MDAC, Jongen ELW (2013) A structural analysis of labour supply elasticities in the Netherlands. CPB Discussion Paper:235

McClelland R, Mok S (2012) A review of recent research on labor supply elasticities. In: Congressional Budget Office. DC, Washington

Meghir C, Phillips D (2008) Labour supply and taxes. The Mirrlees Review: reforming the tax system for the 21st century 
Mroz T (1987) The sensitivity of an empirical model of married women's hours of work to economic and statistical assumptions. Econometrica 55(4):765-799

Orsini K (2012) Tax benefit reforms and the labor market: evidence from Belgium and other EU countries. Labour Econ 19(1):129-138. Previously Leuven University, working paper. 2006

Orsini, K (2007) Ex-ante evaluations of tax and benefit reforms in the EU: what can we learn? Leuven University, working paper

Pencavel J (1986) Labor supply of men: a survey. In: Ashenfelter O, Layard R (eds). Handbook of labor economics. North-Holland, Amsterdam Vol. 1

Pencavel, J (1998) The market work behavior and wages of women, 1975-94. J Hum Resour 33(4):771-804

Pencavel J (2002) A cohort analysis of the association between work hours and wages among men. J Hum Resour 37(2):251-274

Piketty T (1998) L'impact des incitations financières au travail sur les comportements individuels: une estimation pour le cas français. Economie et Pré 132-133:1-35

Prescott EC (2004) Why do Americans work so much more than Europeans? Quarterly Review. Federal Reserve Bank of Minneapolis, issue Jul:2-13

Saez E, Slemrod J, Giertz SH (2012) The elasticity of taxable income with respect to marginal tax rates: a critical review. J Econ Lit 50(1):3-50

Smith N (1995) A panel study of labour supply and taxes in Denmark. Appl Econ 27(5):419-429

Stancanelli E (2008) Evaluating the impact of the French tax credit on the employment rate of women. J Public Econ 92(10-11):2036-47

Steiner V, Wrohlich K (2004) Household taxation, income splitting and labor supply incentives-a microsimulation study for Germany. CESifo Econ Stud 50(3):541-568

Triest RK (1990) The effect of income taxation on labor supply in the United States. J Hum Resour 25:491-516

van Soest A (1995) Structural models of family labor supply: a discrete choice approach. J Hum Resour 30:63-88

van Soest A, Das M (2001) Family labor supply and proposed tax reforms in the Netherlands. De Economist 149(2):191-218

van Soest A, Das M, Gong X (2002) A structural labor supply model with non-parametric preferences. J Econom (Annals) 107:345-374

van Soest A, Woittiez I, Kapteyn A (1990) Labour supply, income taxes and hours restrictions in the Netherlands. J Hum Resour 25:517-558

Walker I (1990) The effect of income support measures on the labour market behaviour of lone mothers. Fisc Stud 11(2):55-74

Wrohlich K (2006) Child care costs and mothers' labor supply: an empirical analysis for Germany. DIW Berlin

Ziliak JP, Kniesner TJ (1999) Estimating life cycle labor supply tax effects. J Polit Econ 107(2):326-59

\section{Submit your manuscript to a SpringerOpen ${ }^{\circ}$ journal and benefit from:}

$\checkmark$ Convenient online submission

- Rigorous peer review

- Immediate publication on acceptance

- Open access: articles freely available online

- High visibility within the field

Retaining the copyright to your article

Submit your next manuscript at $\triangleright$ springeropen.com 